\title{
Values of Brownian intersection exponents, I: Half-plane exponents
}

\author{
by \\ GREGORY F. LAWLER \\ ODED SCHRAMM \\ and \\ WENDELIN WERNER \\ Duke University \\ Durham, NC, U.S.A. \\ Microsoft Research \\ Redmond, WA, U.S.A. \\ Université Paris-Sud \\ Orsay, France \\ and \\ The Weizmann Institute of Science \\ Rehovot, Israel
}

\section{Introduction}

Theoretical physics predicts that conformal invariance plays a crucial role in the macroscopic behavior of a wide class of two-dimensional models in statistical physics (see, e.g., [4], [6]). For instance, by making the assumption that critical planar percolation behaves in a conformally invariant way in the scaling limit, and using ideas involving conformal field theory, Cardy [7] produced an exact formula for the limit, as $N \rightarrow \infty$, of the probability that, in two-dimensional critical percolation, there exists a cluster crossing the rectangle $[0, a N] \times[0, b N]$. Also, Duplantier and Saleur [13] predicted the "fractal dimension" of the hull of a very large percolation cluster. These are just two examples among many such predictions.

In 1988, Duplantier and Kwon [12] suggested that the ideas of conformal field theory can also be applied to predict the intersection exponents between random walks in $\mathbf{Z}^{2}$ (and Brownian motions in $\mathbf{R}^{2}$ ). They predicted, for instance, that if $B$ and $B^{\prime}$ are independent planar Brownian motions (or simple random walks in $\mathbf{Z}^{2}$ ) started from distinct points in the upper half-plane $\mathbf{H}=\{(x, y): y>0\}=\{z \in \mathbf{C}: \operatorname{Im}(z)>0\}$, then when $n \rightarrow \infty$,

$$
\mathbf{P}\left[B[0, n] \cap B^{\prime}[0, n]=\varnothing\right]=n^{-\zeta+o(1)}
$$

and

$$
\mathbf{P}\left[B[0, n] \cap B^{\prime}[0, n]=\varnothing \text { and } B[0, n] \cup B^{\prime}[0, n] \subset \mathbf{H}\right]=n^{-\tilde{\zeta}+o(1)},
$$

where

$$
\zeta=\frac{5}{8}, \quad \tilde{\zeta}=\frac{5}{3}
$$


Very recently, Duplantier [11] gave another physical derivation of these conjectures based on "quantum gravity".

In 1982, Mandelbrot [35] suggested that the Hausdorff dimension of the Brownian frontier (i.e., the boundary of a connected component of the complement of the path) is $\frac{4}{3}$, based on simulations and the analogy with the conjectured value for the fractal dimension of self-avoiding walks predicted by Nienhuis (also $\frac{4}{3}$; see, e.g., [33]).

To date, none of the physicists' arguments have been made rigorous, and it seems very difficult to use their methods to produce proofs. Very recently, Kenyon [16], [17], [18] managed to derive the exact values of critical exponents for "loop-erased random walk" that theoretical physicists had predicted (Majumdar [34], Duplantier [10]). Kenyon's methods involve the relation of the loop-erased walk to the uniform spanning tree and to domino tilings. Kenyon shows that the equations relating probabilities of some domino tiling events are discrete analogues of the Cauchy-Riemann equations, and therefore the probabilities can be approximated by analytic functions with prescribed boundary behavior. These methods do not seem applicable for the goals of the present paper.

For planar Brownian motions, it is easy to show, using subadditivity arguments and the scaling property, that there exist positive finite numbers $\zeta$ and $\tilde{\zeta}$ such that (1.1) and (1.2) are true. Up to the present paper, there was not even a mathematical heuristic arguing that the values of $\zeta$ and $\tilde{\zeta}$ are $\frac{5}{8}$ and $\frac{5}{3}$. Burdzy-Lawler [5] (see also [9], [24]) showed that the intersection exponents were indeed the same for simple random walks as for Brownian motions; Lawler [21] proved that the Hausdorff dimension of the set of cut points of a Brownian path is $2-2 \zeta$. He also showed (see [22], [23]) that the dimension of the Brownian frontier (and more generally the whole multifractal spectrum of the Brownian frontier) can be expressed in terms of exponents defined analogously to $\zeta$. As part of that work, he showed that the right-hand side of (1.1) can be replaced with $n^{-\zeta} g(n)$ where $g$ is bounded away from 0 and infinity; we expect that the argument can be adapted to show that the same is true for (1.2).

Recently, Lawler and Werner [28] extended the definition of intersection exponents in a natural way to "non-integer packets of Brownian motions", and derived certain functional relations between these exponents. These relations indicate that Mandelbrot's conjecture that the dimension of the Brownian frontier is $\frac{4}{3}$ is indeed compatible with the predictions of Duplantier-Kwon. It turned out that intersection exponents in the halfplane play an important role in understanding exponents in the whole plane. Conformal invariance of planar Brownian motion is a crucial tool in the derivation of these relations. In particular, there is a measure on Brownian excursions in domains that has some strong conformal invariance properties, including a "restriction" (or "locality") property.

In another paper, Lawler and Werner [29] showed that intersection exponents as- 
sociated to any conformally invariant measure on sets with this restriction property are very closely related to the Brownian exponents. This provides a rigorous justification to the link between the conjectures regarding intersection exponents for planar Brownian motions and conjectures for intersection exponents of critical percolation clusters (see [13], [8], [3]), because percolation clusters are conjectured to be conformally invariant in the scaling limit-see, e.g., [19], [2] — and they should also have a restriction property (because of the independence properties of percolation). The question of how to compute these exponents remained open.

Independently, Schramm [42] defined a new class of conformally invariant stochastic processes indexed by a real parameter $\kappa \geqslant 0$, called $\mathrm{SLE}_{\kappa}$ (for stochastic Löwner evolution process with parameter $\kappa$ ). The definition of these processes is based on Löwner's ordinary differential equation that encodes in a conformally invariant way a continuous family of shrinking domains (see, e.g., [32], [37]). More precisely, [42] defines a family of conformal maps $g_{t}$ from subsets $D_{t}$ of $\mathbf{H}$ onto $\mathbf{H}$ by the equation

$$
\partial_{t} g_{t}(z)=\frac{-2}{\beta_{\kappa t}-g_{t}(z)}
$$

where $\beta$ is a standard Brownian motion on the real line. (Actually, in [42], instead of (1.3), the corresponding equation for the inverse maps $g_{t}^{-1}$ is considered.) The domain $D_{t}$ can be defined as the set of $z_{0} \in \mathbf{H}$ such that a solution $g_{s}\left(z_{0}\right)$ of this equation exists for $s \in[0, t]$. When $t$ increases, the set $K_{t}=\mathbf{H} \backslash D_{t}$ increases: Loosely speaking, $\left(K_{t}, t \geqslant 0\right)$ can be viewed as a growing "hull" that is penetrating the half-plane. By applying a conformal homeomorphism $f: \mathbf{H} \rightarrow D, \mathrm{SLE}_{\kappa}$ can similarly be defined in any simply-connected domain $D \varsubsetneqq \mathbf{C}$.

In [42], the main focus is on the case $\kappa=2$, which is conjectured there to correspond to the scaling limit of loop-erased random walks, but the conjecture that $\mathrm{SLE}_{6}$ corresponds to the scaling limit of critical percolation cluster boundaries is also mentioned. In particular, it is possible to compute explicitly the probability that an $\mathrm{SLE}_{6}$ crosses a rectangle of size $a \times b$. It turns out that this result is exactly Cardy's formula. This gives a mathematical proof for Cardy's formula, assuming the still open conjecture that $\mathrm{SLE}_{6}$ is indeed the scaling limit of percolation cluster boundaries.

The main goal of the present paper is to prove some of the conjectured values of intersection exponents of Brownian motion in a half-plane.

Theorem 1.1. Let $B^{1}, \ldots, B^{p}$ denote $p$ independent planar Brownian motions $(p \geqslant 2)$ started from distinct points in the upper half-plane $\mathbf{H}$. Then, when $t \rightarrow \infty$,

$$
\mathbf{P}\left[\forall i \neq j \in\{1, \ldots, p\}, B^{i}[0, t] \cap B^{j}[0, t]=\varnothing \text { and } B^{i}[0, t] \subset \mathbf{H}\right]=t^{-\tilde{\zeta}_{p}+o(1)},
$$

where

$$
\tilde{\zeta}_{p}=\frac{1}{6} p(2 p+1)
$$


These values have been predicted by Duplantier and Kwon [12]. In particular, $\tilde{\zeta}=$ $\tilde{\zeta}_{2}=\frac{5}{3}$.

We also establish the exact value (and confirm some of the conjectures stated in [28], [11]) of more general intersection exponents between packets of Brownian motions in the half-plane; see Theorem 4.1.

The proof of Theorem 1.1 uses a combination of ideas from the papers [28], [29], [42]. However, to make the paper more accessible and self-contained, we attempt to review and explain all the necessary background. The reader who wishes to see complete proofs for all stated theorems has to be familiar with the basics of stochastic calculus and conformal mapping theory, and read about the excursion measure and the cascade relations from [28].

Although, at present, a proof of the conjecture that $\mathrm{SLE}_{6}$ is the scaling limit of critical percolation cluster boundaries seems out of reach, this conjecture does lead one to believe that $\mathrm{SLE}_{6}$ must satisfy a "locality" property; namely, it is not affected by the boundary of a domain when it is in the interior. This locality property for $\mathrm{SLE}_{6}$ is stated more precisely and proved in $\S 2$. It is worthwhile to note that the locality property does not hold for the $\mathrm{SLE}_{\kappa}$-processes when $\kappa \neq 6$.

In $\S 3$, we prove that $\mathrm{SLE}_{6}$ satisfies a generalization of Cardy's formula for percolation crossings probabilities. From this, exponents associated with the $\mathrm{SLE}_{6}$-process are computed.

In $\S 4$, universality ideas from [29] are used to compute the half-plane Brownian exponents from the $\mathrm{SLE}_{6}$-exponents, which completes the proof of Theorem 1.1.

In a final short $\S 5$, the conjectured relationship between $\mathrm{SLE}_{6}$ and critical percolation is discussed. It is demonstrated that this conjecture implies a formula from the physics literature [13], [8], [3] for the exponents corresponding to the event that there are $k$ disjoint percolation crossings of a long rectangle.

In the subsequent papers [25], [26], [27], we determine the exponents in the full plane and the remaining half-plane exponents. In particular, we prove that $\zeta=\frac{5}{8}$, and also establish Mandelbrot's conjecture that the Hausdorff dimension of the frontier of planar Brownian motion is $\frac{4}{3}$.

It might be worthwhile to explain why the Brownian intersection exponents are accessible through $\mathrm{SLE}_{6}$, but are difficult to compute directly. In a way, the $\mathrm{SLE}_{6}$ process is simpler, since $K_{t}$ continuously grows from its outer boundary. This means that when studying its evolution, one can essentially forget its interior, and only keep track of the exterior of $K_{t}$. By conformal invariance, this reduces problems to finitely many dimensions. The situation with planar Brownian motion is completely different, since it may enter holes it has surrounded and emerge to the exterior someplace else. 
Many computations with $\mathrm{SLE}_{\kappa}$ are readily convertible to PDE problems, and in the presence of enough symmetry, some variables can often be eliminated, converting the PDE to an ODE.

\section{2. $\mathrm{SLE}_{6}$ and its locality property}

\subsection{The definition of chordal $\mathrm{SLE}_{\kappa}$ and some basic properties}

Let $\left(\beta_{t}, t \geqslant 0\right)$ be a standard real-valued Brownian motion starting at $\beta_{0}=0$, let $\kappa>0$, and let $W_{t}^{\kappa}=\beta_{\kappa t}$. Consider the ordinary differential equation

$$
\partial_{t} g_{t}(z)=\frac{-2}{W_{t}^{\kappa}-g_{t}(z)}
$$

with $g_{0}(z)=z$. For every $z_{0} \in \mathbf{H}$ and every $T>0$, either there is a solution of (2.1) for $t \in[0, T]$ and for all $z$ in a neighborhood of $z_{0}$, or there is some $t_{0} \in(0, T]$ such that the solution exists for $t \in\left[0, t_{0}\right)$ and $\lim _{t} \tau_{0} g_{t}(z)=W_{t_{0}}^{\kappa}$. Let $D_{T}$ be the (open) set of $z \in \mathbf{H}$ such that the former is true, and let $K_{T}$ be the set of $z \in \mathbf{H}$ such that the latter holds. By considering the inverse flow $\partial_{t} G_{t}(z)=2\left(W_{T-t}^{\kappa}-G_{t}(z)\right)^{-1}$, it is easy to see that $g_{t}\left(D_{t}\right)=\mathbf{H}$, and that $g_{t}: D_{t} \rightarrow \mathbf{H}$ is conformal. The process $g_{t}, t \geqslant 0$, will be called the chordal stochastic Löwner evolution process with parameter $\kappa$, or just $\mathrm{SLE}_{\kappa}$; see [42]. In [42], a variation of this process, which we now call radial $\mathrm{SLE}_{\kappa}$ was also studied. In the current paper, we will not use radial $\mathrm{SLE}_{\kappa}$, and therefore the word "chordal" will usually be omitted. (However, radial $\mathrm{SLE}_{\kappa}$ plays a major role in a subsequent paper [25].) The set $K_{t}=\mathbf{H} \backslash D_{t}$ will be called the $h u l l$ of the SLE. The process $W_{t}^{\kappa}$ will be called the driving process of the SLE.

It is easy to verify that each of the maps $g_{t}$ satisfies the hydrodynamic normalization at infinity:

$$
\lim _{z \rightarrow \infty} g(z)-z=0
$$

Remarks. It will be shown [40] that for all $\kappa \neq 8$ the hull $K_{t}$ of $\mathrm{SLE}_{\kappa}$ is a.s. generated by a path. More precisely, a.s. the map $\gamma(t):=g_{t}^{-1}\left(W_{t}^{\kappa}\right)$ is a well-defined continuous path in $\overline{\mathbf{H}}$, and for every $t \geqslant 0$ the domain $D_{t}$ is the unbounded connected component of $\mathbf{H} \backslash \gamma([0, t])$. There, it will also be shown that when $\kappa \leqslant 4$ a.s. $K_{t}$ is a simple path for all $t>0$. This is not the case when $\kappa>4$ [42]. However, these results will not be needed for the current paper or for [25], [26], [27].

Löwner [32] considered the equation

$$
\partial_{t} g_{t}(z)=g_{t}(z) \frac{\zeta(t)+g_{t}(z)}{\zeta(t)-g_{t}(z)}
$$


with $g_{0}(z)=z$, where $z$ is in the unit disk, and $\zeta(t)$ is a parameter. He used this equation in the study of extremal problems for classes of normalized conformal mappings. In Löwner's differential equation, the maps $g_{t}$ satisfies $g_{t}(0)=0$. The equation $(2.1)$ is an analogue of Löwner's equation in the half-plane, where the boundary point $\infty$ is fixed instead of 0 , and $\zeta(t)$ is chosen to be scaled Brownian motion.

Marshall and Rohde [36] study conditions on $\zeta(t)$ which imply that $K_{t}$ is a simple path.

We now note some basic properties of $\mathrm{SLE}_{\kappa}$.

Proposition 2.1. (i) [Scaling] $\mathrm{SLE}_{\kappa}$ is scale-invariant in the following sense. Let $K_{t}$ be the hull of $\mathrm{SLE}_{\kappa}$, and let $\alpha>0$. Then the process $t \mapsto \alpha^{-1 / 2} K_{\alpha t}$ has the same law as $t \mapsto K_{t}$.

(ii) [Stationarity] Let $g_{t}$ be an $\mathrm{SLE}_{\kappa}$-process in $\mathbf{H}$, driven by $W_{t}^{\kappa}$, and let $\tau$ be any stopping time. Set $\tilde{g}_{t}(z)=g_{\tau+t^{\circ}} g_{\tau}^{-1}\left(z+W_{\tau}^{\kappa}\right)-W_{\tau}^{\kappa}$. Then $\tilde{g}_{t}$ is an $\mathrm{SLE}_{\kappa}$-process in $\mathbf{H}$ starting at 0 , which is independent from $\left\{g_{t}: t \in[0, \tau]\right\}$.

Proof. (i) If $K_{t}$ is driven by $W_{t}^{\kappa}$, then $\alpha^{-1 / 2} K_{\alpha t}$ is driven by $\alpha^{-1 / 2} W_{\alpha t}^{\kappa}$, which has the same law as $W_{t}^{\kappa}$.

(ii) The process $\tilde{g}_{t}$ is driven by $W_{t+\tau}^{\kappa}-W_{\tau}^{\kappa}$.

We now consider the definition of $\mathrm{SLE}_{\kappa}$ in domains other than $\mathbf{H}$.

Let $f: D \rightarrow \mathbf{H}$ be a conformal homeomorphism from some simply-connected domain $D$. Let $f_{t}$ be the solution of $(2.1)$ with $f_{0}(z)=f(z)$. Then $\left(f_{t}, t \geqslant 0\right)$ will be called the $\mathrm{SLE}_{\kappa}$ in $D$ starting at $f$. If $g_{t}$ is the solution of $(2.1)$ with $g_{0}(z)=z$, then we have $f_{t}=g_{t} \circ f$. If $K_{t}$ is the hull associated to $g_{t}$, then the hull associated with $f_{t}$ is just $f^{-1}\left(K_{t}\right)$.

Suppose that $\partial D$ is a Jordan curve in $\mathbf{C}$, and let $a, b \in \partial D$ be distinct. Then we may

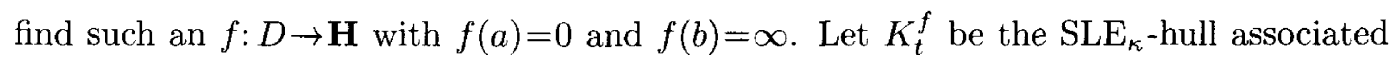
with the $\mathrm{SLE}_{\kappa}$-process starting at $f$. If $f^{*}: D \rightarrow \mathbf{H}$ is another such map with $f^{*}(a)=0$ and $f^{*}(b)=\infty$, then $f^{*}(z)=\alpha f(z)$ for some $\alpha>0$. By Proposition 2.1, the corresponding $\mathrm{SLE}_{\kappa}$-hull $K_{t}^{f^{*}}$ has the same law as a linear time change of $K_{t}^{f}$. This makes it natural to consider $K_{t}^{f}$ as a process from $a$ to $b$ in $D$, and to ignore the role of $f$. However, when $D$ is not a Jordan curve, some care may be needed since the conformal map $f$ does not necessarily extend continuously to the boundary. Partly for that reason, we have chosen to stress the importance of the conformal parameterization $f$. 


\subsection{The locality property}

The main result of this section can be loosely described as follows: an $\mathrm{SLE}_{6}$ process does not feel where the boundary of the domain lies as long as it does not hit it. This is consistent with the conjecture [42] that the $\mathrm{SLE}_{6}$-process is the scaling limit of percolation cluster boundaries, which is explained in $\S 5$. This restriction property can therefore be viewed as additional evidence in favor of this conjecture. This feature is special to $\mathrm{SLE}_{6}$; it is not shared by $\operatorname{SLE}_{\kappa}$ when $\kappa \neq 6$.

Such properties were studied in [29] and called "complete conformal invariance" (when combined with a conformal invariance property). As pointed out there, all processes with complete conformal invariance have closely related intersection exponents.

Let us first state a general local version of this result. We will say that the path $\gamma$ is nice if it is a continuous simple path $\gamma:[0,1] \rightarrow \overline{\mathbf{H}}$ such that $\gamma(0), \gamma(1) \in \mathbf{R} \backslash\{0\}$ and $\gamma(0,1) \subset \mathbf{H}$. We then call the connected component $N=N(\gamma)$ of $\mathbf{H} \backslash \gamma[0,1]$ such that $0 \in \partial N$ a nice neighborhood of 0 in $\mathbf{H}$. Note that $N$ can be bounded or unbounded, depending on the sign of $\gamma(0) \gamma(1)$. When $N$ is a nice neighborhood of 0 , one can find a conformal homeomorphism $\psi=\psi_{N}$ from $N$ onto $\mathbf{H}$ such that $\psi(0)=0, \psi^{\prime}(0)=1$ and $\psi^{-1}(\infty)$ is equal to $\infty$ if $N$ is unbounded and to $\gamma(1)$ if $N$ is bounded.

THEOREM 2.2 (locality). Let $f: D \rightarrow \mathbf{H}$ be a conformal homeomorphism from a domain $D \subset \mathbf{C}$ onto $\mathbf{H}$. Suppose that $N$ is a nice neighborhood of 0 in $\mathbf{H}$. Define $D^{*}=f^{-1}(N)$ and let $f^{*}$ be the conformal homeomorphism $\psi_{N} \circ f$ from $D^{*}$ onto $\mathbf{H}$. Let $K_{t} \subset D$ be the hull of $\mathrm{SLE}_{6}$ starting at $f$, and let $\tau:=\sup \left\{t: \bar{K}_{t} \cap \partial D^{*} \cap D=\varnothing\right\}$. Let $K_{t}^{*}$ denote $\mathrm{SLE}_{6}$ in $D^{*}$ started at $f^{*}$, and let $\tau^{*}:=\sup \left\{t: \bar{K}_{t}^{*} \cap \partial D^{*} \cap D=\varnothing\right\}$.

Then the law of $\left(K_{t}, t<\tau\right)$ is that of a time change of $\left(K_{t}^{*}, t<\tau^{*}\right)$.

Note that in this theorem, we have not made any regularity assumption on the boundary of the domain $D$.

A consequence of this result is that, modulo time change, one can define the hull of $\mathrm{SLE}_{6}$ in a non-simply-connected domain with finitely many boundary components, since such a domain looks locally like a simply-connected domain.

This property implies the following "global" restriction properties. For convenience only, we will state them under some assumptions on the boundaries of the domains.

COROllary 2.3 (splitting property). Let $D$ denote a simply-connected domain such that $\partial D$ is a Jordan curve. Let $a, b$ and $b^{\prime}$ denote three distinct points on $\partial D$, and let $I$ denote the connected component of $\partial D \backslash\left\{b, b^{\prime}\right\}$ that does not contain a. Let $\left(K_{t}, t \geqslant 0\right)$ (resp. $K_{t}^{\prime}$ ) denote an $\mathrm{SLE}_{6}$ in $D$ from a to $b$ (resp. from a to $\left.b^{\prime}\right)$. Let $T$ (resp. $\left.T^{\prime}\right)$ denote the first time at which $\bar{K}_{t}$ (resp. $\left.\bar{K}_{t}^{\prime}\right)$ intersects $I$. Then $\left(K_{t}, t<T\right)$ and $\left(K_{t}^{\prime}, t<T^{\prime}\right)$ have the same law up to time change. 
COROLlaRY 2.4 (restriction property). Let $D^{*} \subset D$ denote two simply-connected domains, and assume that $\partial D$ is a Jordan curve. Suppose that $I:=\partial D^{*} \backslash \partial D$ is connected. Take two distinct points $a$ and $b$ in $\partial D \cap \partial D^{*} \backslash \bar{I}$.

Let $\left(K_{t}, t \geqslant 0\right)$ denote $\mathrm{SLE}_{6}$ from a to $b$ in $D$, and $T:=\sup \left\{t: \bar{K}_{t} \cap I=\varnothing\right\}$. Similarly, let $\left(K_{t}^{*}, t \geqslant 0\right)$ be $\mathrm{SLE}_{6}$ from a to $b$ in $D^{*}$, and $T^{*}:=\sup \left\{t: \bar{K}_{t}^{*} \cap I=\varnothing\right\}$. Then, $\left(K_{t}, t<T\right)$ and $\left(K_{t}^{*}, t<T^{*}\right)$ have the same law up to time change.

In the present paper, we will use these results when $D$ is a rectangle.

Proof of Corollary 2.3 (assuming Theorem 2.2). This is just a consequence of the fact that in Theorem 2.2 with $D=\mathbf{H}$ and bounded $N$, one can replace $\gamma$ by $\beta(s):=\gamma(1-s)$. Then we get that the law of $\mathrm{SLE}_{6}$ in $N$ from 0 to $\gamma(0)$ is that of a time change of $\mathrm{SLE}_{6}$ in $N$ from 0 to $\gamma(1)$ up to their hitting times of $\gamma$. The result in a general domain follows by mapping it conformally onto a nice neighborhood $N$ with $a$ mapped to 0 and $\left\{b, b^{\prime}\right\}$ to $\{\gamma(0), \gamma(1)\}$.

Proof of Corollary 2.4 (assuming Theorem 2.2). By approximation, it suffices to consider the case where $I$ is a simple path. Let $f$ denote a conformal map from $D$ onto $\mathbf{H}$, with $f(a)=0$ and $f(b)=\infty$. Define $\gamma$ in such a way that $\gamma[0,1]=f(I)$; note that $D^{*}=f^{-1}(N(\gamma))$. As $b \in \partial D^{*} \backslash I, N(\gamma)$ is unbounded. Hence, by Theorem 2.2 , the law of $\mathrm{SLE}_{6}$ in $D$ from $a$ to $b$ stopped when it hits $I$ is (up to time change) the same as that of $\mathrm{SLE}_{6}$ in $D^{*}$ from $a$ to $b$ stopped when its closure hits $I$.

In order to prove Theorem 2.2 , we will establish

LEMMA 2.5. Under the assumptions of Theorem 2.2, define for any fixed $s<1$, $L_{s}=\gamma(0, s]$ and

$$
T=\sup \left\{t \geqslant 0: \bar{K}_{t} \cap \bar{L}_{s}=\varnothing\right\} .
$$

For all $t \leqslant T$, let $g_{s, t}$ denote the conformal homeomorphism taking $\mathbf{H} \backslash\left(K_{t} \cup L_{s}\right)$ onto $\mathbf{H}$ with the hydrodynamic normalization. Then, the process $\left(g_{s, t}, t<T\right)$ has the same law as a time change of $\mathrm{SLE}_{6}$ in $\mathbf{H} \backslash L_{s}$ starting at $g_{s, 0}-g_{s, 0}(0)$, up to the time when the closure of its hull intersects $\bar{L}_{s}$.

Proof of Theorem 2.2 (assuming Lemma 2.5). In the setting of the lemma, let

$$
h_{s, t}(z)=\frac{g_{s, t}(z)-g_{s, 0}(0)}{g_{s, 0}^{\prime}(0)} .
$$

By Lemma 2.5 and Proposition 2.1, $t \mapsto h_{s, t}$ has the same law as a time change of SLE 6 starting at $h_{s, 0}$. Note that $h_{s, 0}(0)=0, h_{s, 0}^{\prime}(0)=1$. Hence, it follows easily that for all $z \in N(\gamma)$

$$
\lim _{s \rightarrow 1} h_{s, 0}(z)=\psi_{N}(z)
$$


By continuity, if we let $h_{1, t}=\lim _{s \rightarrow 1} h_{s, t}$, then $t \mapsto h_{1, t}$ has the same law as a time-changed $\mathrm{SLE}_{6}$ (in $N$ ) started from $\psi_{N}$. The proof is completed by noting that the hull of $h_{1, t}$ is $K_{t}$.

The idea in the proof of Lemma 2.5 is to study how the process $g_{s, t}$ changes as $s$ increases. For this, we will need to use some of the properties of solutions to (2.1) where $W^{\kappa}$ is replaced by other continuous functions, and to study how (deterministic) families of conformal maps can be represented in this way with some driving function.

\subsection{Deterministic expanding hulls}

2.3.1. Definition and first properties. If $\left(U_{t}, t \in[0, a]\right)$ is a continuous real-valued function, then the process defined by

$$
\partial_{t} g_{t}(z)=\frac{-2}{U_{t}-g_{t}(z)}
$$

and $g_{0}(z)=z$ will be called the Löwner evolution with driving function $U_{t}$. Note that $g_{t}$ satisfies the hydrodynamic normalization (2.2). Moreover,

$$
g_{t}(z)=z+2 t z^{-1}+a_{2}(t) z^{-2}+\ldots, \quad z \rightarrow \infty
$$

for some functions $a_{j}(t), j=2,3, \ldots$. As above, we let $D_{t} \subset \mathbf{H}$ denote the domain of $g_{t}$, and let $K_{t}:=\mathbf{H} \backslash D_{t}$. $K_{t}$ will be called the expanding hull of the process $g_{t}$.

We now address the question of which processes $K_{t}$ can appear as the expanding hull driven by a continuous function $U_{t}$. We say that a bounded set $K \subset \mathbf{H}$ is a hull if $\mathbf{H} \backslash K$ is open and simply-connected. The Riemann mapping theorem tells us that for each hull $K$, there is a unique conformal homeomorphism $g_{K}: \mathbf{H} \backslash K \rightarrow \mathbf{H}$ which satisfies the hydrodynamic normalization (2.2). Let

$$
A(K)=A\left(g_{K}\right):=\frac{1}{2} \lim _{z \rightarrow \infty} z\left(g_{K}(z)-z\right)
$$

that is, $g(z)=z+2 A(g) z^{-1}+\ldots$ near $\infty$. Observe that $A(K)$ is real, because $g_{K}(x)$ is real when $x \in \mathbf{R}$ and $|x|$ is sufficiently large. Moreover, $A(K) \geqslant 0$, because $\operatorname{Im}\left(z-g_{K}(z)\right)$ is a harmonic function which vanishes at infinity and has non-negative boundary values. Note that

$$
A(g \circ h)=A(g)+A(h)
$$

if $g$ and $h$ satisfy the hydrodynamic normalization. It follows that $A(K) \leqslant A(L)$ when $K \subset L$, since $g_{L}=g_{g_{K}(L \backslash K)}{ }^{\circ} g_{K}$.

The quantity $A(g)$ is similar to capacity, and plays an analogous role for the equation (2.1) as capacity plays for Löwner's equation. 
THEOREM 2.6. Let $\left(K_{t}, t \in[0, a]\right)$ be an increasing family of hulls. Then the following are equivalent:

(1) For all $t \in[0, a], A\left(K_{t}\right)=t$, and for each $\varepsilon>0$ there is a $\delta>0$ such that for each $t \in[0, a-\delta]$ there is a bounded connected set $S \subset \mathbf{H} \backslash K_{t}$ with $\operatorname{diam}(S)<\varepsilon$ and such that $S$ disconnects $K_{t+\delta} \backslash K_{t}$ from infinity in $\mathbf{H} \backslash K_{t}$.

(2) There is some continuous $U:[0, a] \rightarrow \mathbf{R}$ such that $K_{t}$ is driven by $U_{t}$.

In [37] a similar theorem is proved for Löwner's differential equation in the disk.

Note that $K_{t}$ may change discontinuously, in the Hausdorff metric, as $t$ increases. For example, consider $\widehat{K}_{t}:=\{\exp (i s): 0<s \leqslant t\}$ when $t<\pi$, and $\widehat{K}_{\pi}:=\{z \in \mathbf{H}:|z| \leqslant 1\}$ and $\widehat{K}_{t+\pi}:=K_{\pi} \cup(-1,-1+i t], t>0$, say, and let $K_{t}:=\widehat{K}_{\phi(t)}$ where $\phi$ is chosen to satisfy $A\left(K_{\phi(t)}\right)=t$.

LEMMA 2.7. Let $r>0$ and $x_{0} \in \mathbf{R}$, and suppose that $K$ is a hull contained in the disk $\left\{z:\left|z-x_{0}\right|<r\right\}$. Then

$$
\left|g_{K}^{-1}(z)-z+\frac{2 A(K)}{z-x_{0}}\right| \leqslant \frac{C r A(K)}{\left|z-x_{0}\right|^{2}}
$$

for all $z \in \mathbf{H}$ with $\left|z-x_{0}\right|>C r$, where $C>0$ is an absolute constant.

Proof of Lemma 2.7. For notational simplicity, we assume that $x_{0}=0$. Clearly, this does not entail any loss of generality. By approximation, we may assume that $K$ has smooth boundary. Let $I \subset \mathbf{R}$ be the smallest interval in $\mathbf{R}$ containing $\left\{g_{K}(x): x \in \partial K \cap \mathbf{H}\right\}$, and let $f:=g_{K}^{-1}$. Let $f_{I}$ be the restriction of $f$ to $I$. Let $f^{*}$ denote the extension of $f$ to $\mathbf{C} \backslash I$, by Schwarz reflection. The Cauchy formula gives

$$
2 \pi i f^{*}(w)=\int_{|z|=R} \frac{f^{*}(z)}{z-w} d z+\int_{I} \frac{f_{I}(x)-\overline{f_{I}(x)}}{x-w} d x
$$

provided that $R>|w|, R>\max \{|x|: x \in I\}$ and $w \in \mathbf{C} \backslash I$. Since $f^{*}(z)=z-2 A(K) z^{-1}+\ldots$ near $\infty$,

$$
\lim _{R \rightarrow \infty} \int_{|z|=R} \frac{f^{*}(z)}{z-w} d z=\lim _{R \rightarrow \infty} \int_{|z|=R} \frac{z}{z-w} d z=2 \pi i w .
$$

Consequently, we have

$$
f^{*}(w)-w=\frac{1}{\pi} \int_{I} \frac{\operatorname{Im}\left(f_{I}(x)\right)}{x-w} d x .
$$

Multiplying by $w$ and taking $w \rightarrow \infty$ gives

$$
A\left(g_{K}\right)=-A\left(f^{*}\right)=\frac{1}{2 \pi} \int_{I} \operatorname{Im}\left(f_{I}(x)\right) d x
$$


Moreover,

$$
f^{*}(w)-w-2 A\left(f^{*}\right) w^{-1}=\frac{1}{\pi} \int_{I} \operatorname{Im}\left(f_{I}(x)\right)\left(\frac{1}{x-w}+\frac{1}{w}\right) d x
$$

and therefore

$$
\begin{aligned}
\left|f^{*}(w)-w-2 A\left(f^{*}\right) w^{-1}\right| & \leqslant \frac{1}{\pi} \int_{I} \operatorname{Im}\left(f_{I}(x)\right) \sup \left\{\left|(x-w)^{-1}+w^{-1}\right|: x \in I\right\} d x \\
& =-2 A\left(f^{*}\right) \sup \left\{\left|\frac{x}{(x-w) w}\right|: x \in I\right\} .
\end{aligned}
$$

Hence, the proof will be complete once we demonstrate that there is some constant $c_{0}$ such that $I \subset\left[-c_{0} r, c_{0} r\right]$. This is easily done, as follows. Define $G(z):=g_{K}(r z) / r$ for $|z|>1$, and write $G(z)=z+a_{1} z^{-1}+a_{2} z^{-2}+\ldots$. The Area Theorem (see, e.g., [41]) gives $1 \geqslant \sum_{j=1}^{\infty} j\left|a_{j}\right|^{2}$. In particular, $\left|a_{j}\right| \leqslant 1$ for $j \geqslant 1$. Consequently, we have $|G(z)-z| \leqslant 1$ for $|z| \geqslant 2$. By Rouché's theorem (e.g. [41]), it follows that $G(\{|z| \geqslant 2\}) \supset\{|z|>3\}$. Consequently, $g_{K}(\mathbf{H} \backslash K) \supset\{|z|>3 r\}$, which gives $I \subset[-3 r, 3 r]$.

For convenience, we adopt the notation

$$
K_{t, u}:=g_{K_{t}}\left(K_{t+u} \backslash K_{t}\right) .
$$

Proof of Theorem 2.6. We start with (1) implies (2). Let $R:=\sup \left\{|z|: z \in K_{a}\right\}$ and $Q:=\{z \in \mathbf{H}:|z|>R+2\}$. Let $t, \delta, \varepsilon$ and $S$ be as in the statement of the theorem, and let $s \in \partial S$. Suppose that $\varepsilon<1$ and $r \in[\varepsilon, \sqrt{\varepsilon}]$. Then there is an arc $\beta_{r}$ of the circle of radius $r$ about $s$ such that $\beta_{r} \subset \mathbf{H} \backslash K_{t}$ and $K_{t} \cup \mathbf{R} \cup \beta_{r}$ separates $K_{t+\delta} \backslash K_{t}$ from $Q$. It therefore follows that the extremal length of the set of arcs in $\mathbf{H} \backslash K_{t}$ which separate $K_{t+\delta} \backslash K_{t}$ from $Q$ in $\mathbf{H} \backslash K_{t}$ is at most const $/ \log (1 / \varepsilon)$. (For the definition and basic properties of extremal length, see [1], [31]. The terms extremal length and extremal distance have the same meaning.) Since extremal length is invariant under conformal maps, it follows that the extremal length of the set of paths in $\mathbf{H}$ that separate $K_{t, \delta}$ from $g_{K_{t}}(Q)$ is at most const $/ \log (1 / \varepsilon)$. Because the diameter of $g_{K_{t}}(\mathbf{H} \backslash Q)$ is bounded by some function of $R$ (this follows since $g_{K_{t}}$ has the hydrodynamic normalization), we conclude that at least one of these arcs has length less than const $/ \log (1 / \varepsilon)$. Consequently, this is a bound on the diameter of $K_{t, \delta}$. Observe that this bound is uniform for all $t \in[0, a-\delta]$. For each $t<a$, we then define $U_{t}$ to be the point in the intersection $\bigcap_{u>0} \bar{K}_{t, u}$. We have an upper bound on $\operatorname{diam}\left(K_{t, \delta}\right)$ which tends to zero uniformly as $\delta \rightarrow 0$, and therefore $\lim _{\delta \rightarrow 0} g_{K_{t, \delta}}(z)-z=0$ uniformly for $z \in \mathbf{H} \backslash K_{t, \delta}$ and $t \leqslant a-\delta$. This implies that $U_{t}$ is uniformly continuous on $[0, a)$ and can be extended continuously to $[0, a]$.

Now let $z_{0} \in \mathbf{H} \backslash K_{a}$. Then there is some $c>0$ such that $\operatorname{Im}\left(g_{K_{t}}\left(z_{0}\right)\right)>c$ for all $t \in[0, a]$. Lemma 2.7 applied with $K=K_{t, u}, z=g_{K_{t+u}}\left(z_{0}\right)$ and $x_{0}=U_{t+u}$ gives

$$
\frac{g_{K_{t}}\left(z_{0}\right)-g_{K_{t+u}}\left(z_{0}\right)}{u}+\frac{2}{g_{K_{t+u}}\left(z_{0}\right)-U_{t+u}} \rightarrow 0
$$


as $\delta \rightarrow 0$. As $g_{K_{t}}\left(z_{0}\right)$ and $U_{t}$ are continuous in $t$, we may therefore conclude that

$$
\partial_{t} g_{K_{t}}\left(z_{0}\right)=\frac{2}{g_{K_{t}}\left(z_{0}\right)-U_{t}},
$$

which gives (2).

The proof that (2) implies (1) is easy. Let $\varepsilon>0$. Given $0 \leqslant t \leqslant t+u<a$, let $\varrho(t, u):=$ $u+\max \left\{U\left(t^{\prime}\right)-U\left(t^{\prime \prime}\right): t^{\prime}, t^{\prime \prime} \in[t, t+u]\right\}$. Observe that $\operatorname{diam}\left(K_{t, u}\right) \rightarrow 0$ if $\varrho(t, u) \rightarrow 0$, and $\varrho(t, u) \rightarrow 0$ if $u \rightarrow 0$. Consequently, the extremal length of the set of paths separating $K_{t, u}$ from $\{z \in \mathbf{H}:|z|>1\}$ in $\mathbf{H}$ goes to zero as $\varrho(t, u) \rightarrow 0$. This implies that there is a path $\beta$ in this set such that $\operatorname{diam}\left(g_{K_{t}}^{-1}(\beta)\right)<\varepsilon$, provided that $u$ is small. We then just take $S=g_{K_{t}}^{-1}(\beta)$.

2.3.2. Time-modified expanding hulls and restriction. Let $\left(K_{t}, t \in[0, a]\right)$ denote a family of hulls, and suppose that there is a monotone increasing homeomorphism $\phi$ : $[0, a] \rightarrow[0, \hat{a}]$ such that $\left(K_{\phi(t)}, t \in[0, \hat{a}]\right)$ is an expanding hull driven by some function $t \mapsto \widehat{U}_{t}$. If additionally $\phi$ is continuously differentiable in $[0, a]$ and $\phi^{\prime}(t)>0$ for each $t \in[0, a]$, then we call $\left(K_{t}, t \in[0, a]\right)$ a time-modified expanding hull, with driving function $U_{t}:=\widehat{U}_{\phi^{-1}(t)}$. Note that, in this case, $\phi^{-1}(t)=A\left(K_{t}\right)$, and that

$$
\partial_{t} g_{K_{t}}(z)=\frac{2 \partial_{t} A\left(K_{t}\right)}{g_{K_{t}}(z)-U_{t}}
$$

Note that in our terminology, an expanding hull is always a time-modified expanding hull.

LEMMA 2.8. Let $\left(K_{t}, t \in[0, a]\right)$ be a time-modified expanding hull, with driving function $\left(U_{t}, t \in[0, a]\right)$. Let $D$ be a relatively open subset of $\overline{\mathbf{H}}$ which contains $\bar{K}_{a}$, and set $D_{\mathbf{R}}:=D \cap \mathbf{R}$. Let $G: D \rightarrow \overline{\mathbf{H}}$ be conformal in $D \backslash D_{\mathbf{R}}$ and continuous in $D$, and suppose that $G\left(D_{\mathbf{R}}\right) \subset \mathbf{R}$. Then $\left(G\left(K_{t}\right), t \in[0, a]\right)$ is a time-modified expanding hull. Moreover,

$$
\partial_{t} A\left(G\left(K_{t}\right)\right)=G^{\prime}\left(U_{0}\right)^{2} \partial_{t} A\left(K_{t}\right) \quad \text { at } t=0
$$

Proof. We first prove (2.7). The proof will be based on (2.5). Note first that if $K^{\prime}=a K$ then $A\left(K^{\prime}\right)=a^{2} A(K)$. Therefore, we may assume that $G^{\prime}\left(U_{0}\right)=1$. Similarly, with no loss of generality, we assume that $U_{0}=G\left(U_{0}\right)=0$. By the reflection principle, $G$ is analytic in $D$.

Set $\widehat{K}_{t}:=G\left(K_{t}\right)$. Let $I_{t} \subset \mathbf{R}$ be the interval corresponding to $\overline{\partial K_{t} \cap \mathbf{H}}$ under $g_{K_{t}}$, and let $\hat{I}_{t}$ be the interval corresponding to $\overline{\partial \widehat{K}_{t} \cap \mathbf{H}}$ under $g_{\widehat{K}_{t}}$. Let $\varepsilon>0$, and let $D_{\varepsilon}:=\{z \in D$ : $\left.\left|1-G^{\prime}(z)\right|<\varepsilon\right\}$. Let $\beta$ be some arc in $D_{\varepsilon} \backslash\{0\}$ that separates 0 from $\infty$ in $\overline{\mathbf{H}}$. Consider the map

$$
h_{t}=g_{\hat{K}_{t}} \circ G \circ g_{K_{t}}^{-1}
$$


It is well defined in a neighborhood of $I_{t}$ provided that $K_{t} \cap \beta=\varnothing$ (for instance), and this holds when $t$ is small. This map may be continued analytically by reflecting in the real axis, and therefore the maximum principle implies that

$$
\sup \left\{h_{t}^{\prime}(x): x \in I_{t}\right\} \leqslant \sup \left\{\left|h_{t}^{\prime}(z)\right|: z \in g_{K_{t}}(\beta)\right\}
$$

when $K_{t} \cap \beta=\varnothing$. Note that $g_{K_{t}}(z)-z \rightarrow 0$ and $g_{\widehat{K}_{t}}(z)-z \rightarrow 0$ as $t \searrow 0$, and therefore $g_{K_{t}}^{\prime}(z) \rightarrow 1$ and $g_{\widehat{K}_{t}}^{\prime}(z) \rightarrow 1$ on $\beta$. Consequently, for small $t$ we have

$$
\sup \left\{h_{t}^{\prime}(x): x \in I_{t}\right\}<1+2 \varepsilon
$$

Note that for $z$ close to $U_{0}=0$ we have $\operatorname{Im}(G(z)) \leqslant(1+\varepsilon) \operatorname{Im}(z)$. Using $(2.5)$, this inequality and (2.8), we get

$$
\begin{aligned}
A\left(G\left(K_{t}\right)\right) & =\frac{1}{2 \pi} \int_{\hat{I}_{t}} \operatorname{Im}\left(g_{\hat{K}_{t}}^{-1}(x)\right) d x \\
& =\frac{1}{2 \pi} \int_{I_{t}} \operatorname{Im}\left(G \circ g_{K_{t}}^{-1}(x)\right) h_{t}^{\prime}(x) d x \\
& \leqslant \frac{1}{2 \pi} \int_{I_{t}}(1+\varepsilon) \operatorname{Im}\left(g_{K_{t}}^{-1}(x)\right)(1+2 \varepsilon) d x \\
& =(1+\varepsilon)(1+2 \varepsilon) A\left(K_{t}\right)
\end{aligned}
$$

for small $t>0$. (Note that $g_{K_{t}}^{-1}(x)$ is not defined for every $x \in I_{t}$, but it is defined for almost every $x \in I_{t}$.) By symmetry, we also have a similar inequality in the other direction. This proves (2.7).

By Theorem 2.6, to show that $G\left(K_{t}\right)$ is a time-modified expanding hull, it suffices to show that $A\left(G\left(K_{t}\right)\right)$ is continuously differentiable in $t$, with derivative bounded away from 0 . Let $G_{t}:=g_{\widehat{K}_{t}}{ }^{\circ} \circ \circ g_{K_{t}}^{-1}$. Then $G_{t}$ is analytic in $g_{K_{t}}\left(D \backslash K_{t}\right)$ and depends continuously on $t$. Hence $G_{t}^{\prime}\left(U_{t}\right)$ is continuous in $t$. Since $A\left(G\left(K_{t+u}\right)\right)=A\left(G\left(K_{t}\right)\right)+A\left(G_{t}\left(K_{t, u}\right)\right)$, it follows that $\partial_{t} A\left(G\left(K_{t}\right)\right)=G_{t}^{\prime}\left(U_{t}\right)^{2} \partial_{t} A\left(K_{t}\right)$, which completes the proof.

For future reference, we note that when $g_{t}=g_{K_{t}}$ satisfies the differential equation (2.6), we have the formula

$$
\partial_{t} \log g_{t}^{\prime}(z)=-\frac{2 \partial_{t} A\left(g_{t}\right)}{\left(g_{t}(z)-U_{t}\right)^{2}}
$$

which is obtained by differentiating (2.6) with respect to $z$. 
2.3.3. Pairs of time-modified expanding hulls. We now discuss the situation where there are two disjoint expanding hulls.

Let $\left(L_{s}, s \in\left[0, s_{0}\right]\right)$ and $\left(K_{t}, t \in\left[0, t_{0}\right]\right)$ be a pair of time-modified expanding hulls such that $\bar{L}_{s_{0}} \cap \bar{K}_{t_{0}}=\varnothing$. Let $g_{s, t}:=g_{L_{s} \cup K_{t}}, g_{t}:=g_{K_{t}}, \hat{g}_{s}:=g_{L_{s}}$ and $a(s, t):=A\left(g_{s, t}\right)$. Then for each $s \in\left[0, s_{0}\right]$ and $t \in\left[0, t_{0}\right]$ we have

$$
g_{s, t}=g_{g_{t}\left(L_{s}\right)^{\circ}} g_{t}
$$

Therefore,

$$
\partial_{s} g_{s, t}(z)=\frac{2 \partial_{s} a(s, t)}{g_{s, t}(z)-U^{1}(s, t)}
$$

where $s \mapsto U^{1}(s, t)$ is the driving function for the time-modified expanding hulls $s \mapsto g_{t}\left(L_{s}\right)$. Similarly,

$$
\partial_{t} g_{s, t}(z)=\frac{2 \partial_{t} a(s, t)}{g_{s, t}(z)-U^{2}(s, t)}
$$

where $t \mapsto U^{2}(s, t)$ is the driving function for the time-modified expanding hulls $t \mapsto \hat{g}_{s}\left(K_{t}\right)$. Although we do not know that $g_{t}^{-1}\left(U^{2}(0, t)\right)$ is well defined, $g_{s, t} \circ g_{t}^{-1}$ is analytic in a neighborhood of $U^{2}(0, t)$, by the reflection principle. Hence, it is clear that $U^{2}(s, t)=$ $g_{s, t^{\circ}} g_{t}^{-1}\left(U^{2}(0, t)\right)$ (see, for example, the construction of $U_{t}$ in the proof of Theorem 2.6), and therefore

$$
\partial_{s} U^{2}(s, t)=\frac{2 \partial_{s} a(s, t)}{U^{2}(s, t)-U^{1}(s, t)}
$$

We will now prove the formula

$$
\partial_{s} \partial_{t} a(s, t)=\frac{-4 \partial_{s} a(s, t) \partial_{t} a(s, t)}{\left(U^{2}(s, t)-U^{1}(s, t)\right)^{2}}
$$

From (2.7) we have

$$
\partial_{t} a(0, s)=\hat{g}_{s}^{\prime}\left(U^{2}(0,0)\right)^{2} \partial_{t} a(0,0)=\hat{g}_{s}^{\prime}\left(U^{2}(0,0)\right)^{2},
$$

and using (2.9), we obtain

$$
\partial_{s} \log \partial_{t} a(0, s)=\frac{-4 \partial_{s} A\left(\hat{g}_{s}\right)}{\left(\hat{g}_{s}\left(U^{2}(0,0)\right)-U^{1}(s, 0)\right)^{2}}=\frac{-4 \partial_{s} a(0, s)}{\left(U^{2}(s, 0)-U^{1}(s, 0)\right)^{2}}
$$

This verifies (2.11) for the case $t=0$. The general case is similarly obtained. 


\subsection{Proof of Lemma 2.5}

We will now prove Lemma 2.5; this is the core of the proof of the locality property. We assume that $\gamma:\left[0, s_{1}\right] \rightarrow \overline{\mathbf{H}}$ is a continuous simple path with $\gamma(0) \in \mathbf{R} \backslash\{0\}$ and $L_{s}:=$ $\gamma\left(0, s_{1}\right] \subset \mathbf{H}$. With no loss of generality, assume that $\gamma$ is parameterized so that $A\left(L_{s}\right)=s$. By Theorem 2.6, $\left(L_{s}, s \in\left[0, s_{1}\right]\right)$ is a time-modified expanding hull, and by Lemma 2.8, for each $s, t \mapsto g_{L_{s}}\left(K_{t}\right)$ is a time-modified expanding hull, and for each $t, s \mapsto g_{K_{t}}\left(L_{s}\right)$ is a time-modified expanding hull. Let $t \mapsto W(s, t)$ be the process driving $t \mapsto g_{L_{s}}\left(K_{t}\right)$, let $U(s, t)$ be the process driving $s \mapsto g_{K_{t}}\left(L_{s}\right)$, and let $Y_{t}=W(0, t)$ be the process driving $K_{t}$. As above, let $a(s, t)=A\left(g_{s, t}\right)$. For simplicity, $W(s, t)$ will be abbreviated to $W, U(s, t)$ to $U, a(s, t)$ to $a$, etc.

Our aim is to show that $\left(W\left(s_{1}, t\right), t \geqslant 0\right)$ is a continuous martingale (up to the stopping time $T$ ), and that its quadratic variation (for background on stochastic calculus, see, e.g., [15], [39]) is

$$
\left\langle W\left(s_{1}, \cdot\right)\right\rangle_{t}=6\left(a\left(s_{1}, t\right)-a\left(s_{1}, 0\right)\right) .
$$

Indeed, if this is true, let $\phi(t)$ be the inverse of the map $t \mapsto a\left(s_{1}, t\right)-a\left(s_{1}, 0\right)$, and define $\widetilde{W}(t)=W\left(s_{1}, \phi(t)\right)$. Then $\widetilde{W}\left(\frac{1}{6} t\right)$ is a Brownian motion, so that $t \mapsto g_{s_{1}, 0}\left(K_{\phi(t)}\right)-g_{s_{1}, 0}(0)$ is an $\mathrm{SLE}_{6}$-process, as required. Note that this will in fact give a precise expression for the time change in Lemma 2.5 and Theorem 2.2.

Before giving the mathematically rigorous proof, we first present a formal, nonrigorous derivation of the fact that $W(s, \cdot)$ is a martingale. In this derivation, $\kappa$ will be kept as a variable, in order to stress where the assumption $\kappa=6$ plays a role (it will not be so apparent in our proof).

Non-rigorous argument. The first goal is to show that the quadratic variation $\langle W\rangle_{t}$ of $t \mapsto W(s, t)$ satisfies

$$
\partial_{t}\langle W\rangle_{t}=\kappa \partial_{t} a
$$

for each $s, t$. It is clear that this holds when $s=0$, since $K_{t}$ is $\mathrm{SLE}_{6}$. We have

$$
\begin{array}{rlrl}
\partial_{s} \partial_{t}\langle W\rangle_{t} & =\partial_{t} \partial_{s}\langle W\rangle_{t} & \\
& =2 \partial_{t}\left\langle\partial_{s} W, W\right\rangle_{t} & \\
& =2 \partial_{t}\left\langle 2\left(\partial_{s} a\right)(W-U)^{-1}, W\right\rangle_{t} & & (\text { by }(2.10)) \\
& =-4\left(\partial_{s} a\right)(W-U)^{-2} \partial_{t}\langle W\rangle_{t} & & \text { (by Itô's formula) } \\
& =\left(\partial_{t} a\right)^{-1}\left(\partial_{s} \partial_{t} a\right) \partial_{t}\langle W\rangle_{t} & & \text { (by (2.11)). }
\end{array}
$$

Consequently,

$$
\left(\partial_{t} a\right)^{2} \partial_{s}\left(\partial_{t}\langle W\rangle_{t} / \partial_{t} a\right)=\partial_{t} a \partial_{s} \partial_{t}\langle W\rangle_{t}-\partial_{t}\langle W\rangle_{t} \partial_{s} \partial_{t} a=0
$$


which means that $\partial_{t}\langle W\rangle_{t} / \partial_{t} a$ does not depend on $s$. Since (2.12) holds when $s=0$, this proves (2.12).

We now show that $t \mapsto W(s, t)$ is a martingale. The $d t$-term in Itô's formula for the $\partial_{t}$-derivative of

$$
\partial_{s} W(s, t)=\frac{2 \partial_{s} a}{W-U}
$$

is

$$
\frac{2 \partial_{t} \partial_{s} a}{W-U}+2 \frac{\partial_{s} a}{(W-U)^{3}} \partial_{t}\langle W\rangle_{t}-4 \frac{\partial_{s} a}{(W-U)^{3}} \partial_{t} a
$$

where the first summand comes from differentiating $\partial_{s} a$, the second summand is the diffusion term in Itô's formula, and the last summand comes from differentiating with respect to $U$ and using (2.10) for $\partial_{t} U$. Using (2.11) and (2.12), this becomes

$$
\left(3-\frac{1}{2} \kappa\right) \frac{\partial_{t} \partial_{s} a}{W-U}
$$

which vanishes when $\kappa=6$. Hence $t \mapsto \partial_{s} W(s, t)$ is a martingale. As

$$
W(s, t)=Y_{t}+\int_{0}^{s} \partial_{s} W\left(s^{\prime}, t\right) d s^{\prime},
$$

it follows that $t \mapsto W(s, t)$ is a martingale. This completes the informal proof.

The problem with the above argument is that we do not know that $t \mapsto W(s, t)$ is a semi-martingale, and hence cannot apply stochastic calculus to it. Moreover, we need to check that there is sufficient regularity to justify the equality $\partial_{s} \partial_{t}\langle W\rangle_{t}=\partial_{t} \partial_{s}\langle W\rangle_{t}$.

To rectify the situation, set

$$
V\left(s, t^{\prime}\right):=W(s, 0)+\int_{0}^{t^{\prime}} \sqrt{\partial_{t} a(s, t)} d Y_{t}
$$

Then $t \mapsto V(s, t)$ is clearly a martingale. The rest of this subsection will be devoted to the proof of the fact that $V=W$. Recall that

$$
T=\sup \left\{t \geqslant 0: \bar{K}_{t} \cap \bar{L}_{s_{1}}=\varnothing\right\}
$$

We will need the following fact:

Lemma 2.9. There exists a continuous version of $V$ on $\left[0, s_{1}\right] \times[0, T)$.

Proof. In order to keep some quantities bounded, we have to stop the processes slightly before $T$. Let us fix $\varepsilon \in(0,1)$, and define

$$
T_{1}^{\varepsilon}=\inf \left\{t>0: \inf _{s \leqslant s_{1}}|W(s, t)-U(s, t)| \leqslant \varepsilon\right\}
$$


Define for any $s \leqslant s_{1}$ and $t_{0} \geqslant 0$

$$
\widetilde{V}\left(s, t_{0}\right)=\widetilde{V}^{\varepsilon}\left(s, t_{0}\right):=\int_{0}^{\min \left(t_{0}, T_{1}^{\varepsilon}\right)} \sqrt{\partial_{t} a} d Y_{t}
$$

As

$$
\sup _{n \geqslant 1} T_{1}^{1 / n}=T
$$

it is sufficient to show existence of a continuous version (on $\left[0, s_{1}\right] \times \mathbf{R}_{+}$) of $\tilde{V}$.

Let

$$
\tilde{a}=\tilde{a}(s, t):=1_{\left\{t \leqslant T_{1}^{\varepsilon}\right\}} \sqrt{\partial_{t} a} .
$$

Note that $\partial_{t} a(0, t)=\partial_{s} a(s, 0)=1$. Hence, from (2.11) it follows that $\partial_{s} a \leqslant 1$ and $\partial_{t} a \leqslant 1$ for all $s \leqslant s_{1}, t<T$. Using (2.11) again, we get

$$
\left|\partial_{s} \tilde{a}\right|=1_{\left\{t \leqslant T_{1}^{\varepsilon}\right\}} \frac{2 \sqrt{\partial_{t} a} \partial_{s} a}{(W-U)^{2}} \leqslant 2 \varepsilon^{-2} .
$$

Hence, for all $t \geqslant 0$, for all $s, s^{\prime}$ in $\left[0, s_{1}\right]$,

$$
\left|\tilde{a}(s, t)-\tilde{a}\left(s^{\prime}, t\right)\right| \leqslant 2 \varepsilon^{-2}\left|s-s^{\prime}\right| .
$$

But

$$
\mathbf{E}\left[\left(\tilde{V}\left(s, t_{0}\right)-\tilde{V}\left(s^{\prime}, t_{0}^{\prime}\right)\right)^{4}\right] \leqslant 16 \mathbf{E}\left[\left(\int_{t_{0}}^{t_{0}^{\prime}} \tilde{a}\left(s^{\prime}, t\right) d Y_{t}\right)^{4}\right]+16 \mathbf{E}\left[\left(\int_{0}^{t_{0}}\left(\tilde{a}(s, t)-\tilde{a}\left(s^{\prime}, t\right)\right) d Y_{t}\right)^{4}\right]
$$

and using, for instance, the Burkholder-Davis-Gundy inequality for $p=4$ (see, e.g., $\left[39\right.$, IV.4]), we see that there exists a constant $c_{1}=c_{1}(\varepsilon)$ such that for all $t_{0}, t_{0}^{\prime} \geqslant 0$ and $s, s^{\prime} \in\left[0, s_{1}\right]$,

$$
\begin{aligned}
\mathbf{E}\left[\left(\tilde{V}\left(s, t_{0}\right)-\tilde{V}\left(s^{\prime}, t_{0}^{\prime}\right)\right)^{4}\right] & \leqslant c_{1} \mathbf{E}\left[\left(t_{0}-t_{0}^{\prime}\right)^{2}\right]+c_{1} \mathbf{E}\left[\left(\int_{0}^{t_{0}}\left(s-s^{\prime}\right)^{2} d t\right)^{2}\right] \\
& \leqslant c_{1}\left(t_{0}-t_{0}^{\prime}\right)^{2}+c_{1} t_{0}^{2}\left(s-s^{\prime}\right)^{4}
\end{aligned}
$$

and the existence of a continuous version of $\widetilde{V}$ then easily follows from Kolmogorov's lemma (see, e.g., [39, I.(1.8)]).

From now on, we will use a version of $V$ that is continuous on $\left[0, s_{1}\right] \times[0, T)$. Define

$$
\begin{aligned}
& T_{2}^{\varepsilon}:=\inf \left\{t \geqslant 0: \sup _{s \leqslant s_{1}}|V(s, t)-W(s, t)| \geqslant 1\right\}, \\
& T_{3}^{\varepsilon}:=\inf \left\{t \geqslant 0: \inf _{s \leqslant s_{1}}|V(s, t)-U(s, t)| \leqslant \varepsilon\right\}, \\
& T^{\varepsilon}:=\min \left(T_{1}^{\varepsilon}, T_{2}^{\varepsilon}, T_{3}^{\varepsilon}\right) .
\end{aligned}
$$


Note that for all $s \leqslant s_{1}$ and $t<T$,

$$
\partial_{s} \sqrt{\partial_{t} a}=\frac{-2 \sqrt{\partial_{t} a} \partial_{s} a}{(W-U)^{2}}
$$

The process $\partial_{s} \sqrt{\partial_{t} a}$ remains bounded before $T^{\varepsilon}$ (uniformly in $s \leqslant s_{1}$ ), and it is a measurable function of $(s, t)$. By Fubini's theorem for stochastic integrals ([15, Lemma III.4.1]), we have that for all $s_{0} \leqslant s_{1}$, for all $t_{0} \geqslant 0$, almost surely

$$
\begin{aligned}
\int_{0}^{s_{0}} \int_{0}^{t_{0}^{\prime}} \frac{-2 \sqrt{\partial_{t} a} \partial_{s} a}{(W-U)^{2}} d Y_{t} d s & =\int_{0}^{t_{0}^{\prime}}\left(\int_{0}^{s_{0}} \partial_{s} \sqrt{\partial_{t} a} d s\right) d Y_{t} \\
& =\int_{0}^{t_{0}^{\prime}}\left(\sqrt{\partial_{t} a\left(s_{0}, t\right)}-\sqrt{\partial_{t} a(0, t)}\right) d Y_{t} \\
& =V\left(s_{0}, t_{0}^{\prime}\right)-V\left(0, t_{0}^{\prime}\right)-\left(W\left(s_{0}, 0\right)-W(0,0)\right)
\end{aligned}
$$

where $t_{0}^{\prime}:=\min \left(t_{0}, T^{\varepsilon}\right)$. On the other hand, using Itô's formula, we now compute

$$
\begin{aligned}
\frac{2 \partial_{s} a\left(s, t_{0}^{\prime}\right)}{U\left(s, t_{0}^{\prime}\right)-V\left(s, t_{0}^{\prime}\right)}= & \frac{2}{U(s, 0)-V(s, 0)}+\int_{0}^{t_{0}^{\prime}} \frac{2 \partial_{s} a \sqrt{\partial_{t} a}}{(U-V)^{2}} d Y_{t} \\
& +2 \int_{0}^{t_{0}^{\prime}}\left(\frac{\partial_{t} \partial_{s} a}{U-V}-\frac{\partial_{s} a \partial_{t} U}{(U-V)^{2}}+\frac{\partial_{s} a \partial_{t}\langle V\rangle_{t}}{(U-V)^{3}}\right) d t \\
= & -\partial_{s} W(s, 0)+\int_{0}^{t_{0}^{\prime}} \tilde{b}_{1} d Y_{t}+\int_{0}^{t_{0}^{\prime}}(V-W) b_{2} d t,
\end{aligned}
$$

where (using $\partial_{t}\langle V\rangle_{t}=\partial_{t} a \partial_{t}\langle Y\rangle_{t}=\kappa \partial_{t} a=6 \partial_{t} a$ )

$$
\begin{aligned}
& \tilde{b}_{1}(s, t):=\frac{2 \partial_{s} a \sqrt{\partial_{t} a}}{(U-V)^{2}}, \\
& b_{2}(s, t):=\frac{4 \partial_{s} a \partial_{t} a}{(U-W)^{2}(U-V)^{2}}\left(5+3 \frac{W-V}{V-U}\right) .
\end{aligned}
$$

Note that for all $s \leqslant s_{1}$ and $t \leqslant T^{\varepsilon}$,

$$
\left|b_{2}(s, t)\right| \leqslant 16 \varepsilon^{-5}
$$

By integrating (2.14) with respect to $s$ and subtracting (2.13) from it, we get

$$
\begin{aligned}
V\left(s_{0}, t_{0}^{\prime}\right)-V\left(0, t_{0}^{\prime}\right)+\int_{0}^{s_{0}} \frac{2 \partial_{s} a\left(s, t_{0}^{\prime}\right)}{U\left(s, t_{0}^{\prime}\right)-V\left(s, t_{0}^{\prime}\right)} d s \\
=\int_{0}^{s_{0}} \int_{0}^{t_{0}^{\prime}}(V-W) b_{2}(s, t) d t d s \\
\quad+\int_{0}^{s_{0}}\left(\int_{0}^{t_{0}^{\prime}} \frac{-2 \sqrt{\partial_{t} a} \partial_{s} a}{(W-U)^{2}} d Y_{t}+\int_{0}^{t_{0}} \tilde{b}_{1}(s, t) d Y_{t}\right) d s \\
=\int_{0}^{s_{0}} \int_{0}^{t_{0}^{\prime}}(V-W) b_{2} d t d s+\int_{0}^{s_{0}} \int_{0}^{t_{0}^{\prime}}(V-W) b_{1} d Y_{t} d s
\end{aligned}
$$


where (after some simplifications)

$$
b_{1}(s, t):=\frac{2 \partial_{s} a \sqrt{\partial_{t} a}}{(V-U)^{2}(W-U)^{2}}((U-W)+(U-V)) .
$$

Note that for all $s \in\left[0, s_{1}\right]$ and $t \leqslant T^{\varepsilon}$,

$$
\left|b_{1}(s, t)\right| \leqslant 4 \varepsilon^{-3}
$$

But we know on the other hand that

$$
W\left(s_{0}, t_{0}^{\prime}\right)-W\left(0, t_{0}^{\prime}\right)=\int_{0}^{s_{0}} \frac{2 \partial_{s} a\left(s, t_{0}^{\prime}\right)}{W\left(s, t_{0}^{\prime}\right)-U\left(s, t_{0}^{\prime}\right)} d s .
$$

Subtracting this equation from (2.15), one gets

$$
\begin{aligned}
V\left(s_{0}, t_{0}^{\prime}\right)-W\left(s_{0}, t_{0}^{\prime}\right)= & \int_{0}^{s_{0}} b_{3}\left(s, t_{0}^{\prime}\right)\left(V\left(s, t_{0}^{\prime}\right)-W\left(s, t_{0}^{\prime}\right)\right) d s \\
& +\int_{0}^{s_{0}} \int_{0}^{t_{0}^{\prime}}(V-W) b_{2} d t d s+\int_{0}^{s_{0}} \int_{0}^{t_{0}^{\prime}}(V-W) b_{1} d Y_{t} d s
\end{aligned}
$$

where

$$
b_{3}(s, t):=\frac{-2 \partial_{s} a}{(U-W)(U-V)} .
$$

Again $b_{3}$ remains uniformly bounded before $T^{\varepsilon}$.

We now define

$$
H(s, t)=V(s, t)-W(s, t)
$$

Hence, for all $t_{0} \leqslant T$,

$$
H\left(s_{0}, t_{0}\right)=\int_{0}^{s_{0}} b_{3}\left(s, t_{0}\right) H\left(s, t_{0}\right) d s+\int_{0}^{s_{0}} \int_{0}^{t_{0}} b_{2} H d s d t+\int_{0}^{s_{0}} \int_{0}^{t_{0}} b_{1} H d s d Y_{t}
$$

and $\left|b_{1}\right|,\left|b_{2}\right|,\left|b_{3}\right|$ are all bounded by some constant $c_{2}=c_{2}(\varepsilon)$ on $\left[0, s_{1}\right] \times\left[0, T^{\varepsilon}\right]$. This equation and an argument similar to Gronwall's lemma will show that $H=0$.

Let us fix $t_{1}>0$. For any $t \geqslant 0$, define

$$
\tau(t)=\min \left(t, t_{1}, T^{\varepsilon}\right)
$$

We will use the notation $\tau_{0}=\tau\left(t_{0}\right)$. It is easy to see that there exists a $c_{3}=c_{3}\left(\varepsilon, t_{1}, s_{1}\right)$ such that for all $t_{0} \geqslant 0$ and $s_{0} \in\left[0, s_{1}\right]$,

$$
H\left(s_{0}, \tau_{0}\right)^{2} \leqslant c_{3} \int_{0}^{s_{0}} H\left(s, \tau_{0}\right)^{2} d s+c_{3} \int_{0}^{\tau_{0}} \int_{0}^{s_{0}} H^{2} d s d t+c_{3}\left(\int_{0}^{\tau_{0}} \int_{0}^{s_{0}} b_{1} H d s d Y_{t}\right)^{2}
$$


The Burkholder-Davis-Gundy inequality for $p=2$ (see, e.g., [39, IV.4]) shows that there exist constants $c_{4}=c_{4}\left(\varepsilon, t_{1}, s_{1}\right)$ and $c_{5}=c_{5}\left(\varepsilon, t_{1}, s_{1}\right)$ such that for all $s \in\left[0, s_{1}\right]$ and $t_{0} \geqslant 0$,

$$
\begin{aligned}
\mathbf{E}\left[\sup _{u \leqslant t_{0}}\left(\int_{0}^{\tau(u)} \int_{0}^{s_{0}} b_{1} H d s d Y_{t}\right)^{2}\right] & \leqslant c_{4} \mathbf{E}\left[\int_{0}^{\tau_{0}}\left(\int_{0}^{s_{0}} b_{1} H d s\right)^{2} d t\right] \\
& \leqslant c_{5} \int_{0}^{t_{0}} \int_{0}^{s_{0}} \mathbf{E}\left[H(s, \tau(t))^{2}\right] d s d t
\end{aligned}
$$

Let us now define

$$
h\left(s_{0}, t_{0}\right)=\mathbf{E}\left[\sup _{t \leqslant t_{0}} H\left(s_{0}, \tau(t)\right)^{2}\right]
$$

Then

$$
h\left(s_{0}, t_{0}\right) \leqslant c_{6}\left(\int_{0}^{s_{0}} h\left(s, t_{0}\right) d s+\int_{0}^{t_{0}} \int_{0}^{s_{0}} h(s, t) d s d t\right) .
$$

We also know that $h(s, t)$ is bounded by 1 (because $|H| \leqslant 1$ ). Hence, it is straightforward to prove by induction that for all $s_{0} \in\left[0, s_{1}\right], t_{0} \geqslant 0$ and $p=1,2, \ldots$,

$$
h\left(s_{0}, t_{0}\right) \leqslant \frac{c_{6}^{p} s_{0}^{p}\left(1+t_{0}\right)^{p}}{p !},
$$

so that $h\left(s_{0}, t_{0}\right)=0$. In particular (using the continuity of $V$ and $W$ ), this shows that $W=V$ almost surely on all sets $\left[0, s_{1}\right] \times\left[0, \min \left(t_{1}, T^{\varepsilon}\right)\right]$. As this is true for all $\varepsilon$ and $t_{1}$, we conclude that $V=W$ on $\left[0, s_{1}\right] \times[0, T)$. Lemma 2.5 follows, and thereby also Theorem 2.2 .

\section{Exponents for $\mathrm{SLE}_{6}$}

\subsection{Statement}

In the present section, we are going to compute intersection exponents associated with $\mathrm{SLE}_{6}$.

Suppose that $D \subset \mathbf{C}$ is a Jordan domain; that is, $\partial D$ is a simple closed curve in $\mathbf{C}$. Let $a, b \in \partial D$ be two distinct points on the boundary of $D$. As explained in $\S 2.1$, the $\operatorname{SLE}_{6}\left(K_{t}, t \geqslant 0\right)$ from $a$ to $b$ in $D$ is well defined, up to a linear time change.

Now suppose that $I \subset \partial D$ is an arc with $b \in I$ but $a \notin I$. Let

$$
\tau_{I}:=\sup \left\{t \geqslant 0: \bar{K}_{t} \cap I=\varnothing\right\} .
$$

By Corollary 2.3, up to a time change, the law of the process $\left(K_{t}, t<\tau_{I}\right)$ does not change if we replace $b$ by another point $b^{\prime} \in I$. Set

$$
S=S(a, I, D):=\bigcup_{t<\tau_{I}} K_{t}
$$


and call this set the hull from a to $I$ in $D$. It does not depend on $b$.

Suppose that $L>0$, and let $\mathcal{R}=\mathcal{R}(L)$ denote the rectangle with corners

$$
A_{1}:=0, \quad A_{2}:=L, \quad A_{3}:=L+i \pi, \quad A_{4}:=i \pi .
$$

Let $\mathcal{S}$ denote the closure of the hull from $A_{4}$ to $\left[A_{1}, A_{2}\right] \cup\left[A_{2}, A_{3}\right]$ in $\mathcal{R}$.

In the following, we will use the terminology $\pi$-extremal distance instead of " $\pi$ times the extremal distance". For instance, the $\pi$-extremal distance between the vertical sides of $\mathcal{R}$ in $\mathcal{R}$ is $L$.

When $\mathcal{S} \cap\left[A_{1}, A_{2}\right]=\varnothing$, let $\mathcal{L}$ be the $\pi$-extremal distance between $\left[A_{1}, A_{4}\right]$ and $\left[A_{2}, A_{3}\right]$ in $\mathcal{R} \backslash \mathcal{S}$. Otherwise, put $\mathcal{L}=\infty$.

In the sequel, we will use the function

$$
u(\lambda)=\frac{1}{6}(6 \lambda+1+\sqrt{24 \lambda+1})
$$

The main goal of this section is to prove the following result.

THEOREM 3.1.

$$
\mathbf{E}\left[1_{\{\mathcal{L}<\infty\}} \exp (-\lambda \mathcal{L})\right]=\exp (-u(\lambda) L+O(1)(\lambda+1)) \quad \text { as } L \rightarrow \infty
$$

for any $\lambda \geqslant 0$ (where $O(1)$ denotes an arbitrary quantity whose absolute value is bounded by a constant which does not depend on $L$ or $\lambda$ ).

In particular, when $\lambda=0$,

$$
\mathbf{P}\left[\mathcal{S} \cap\left[A_{1}, A_{2}\right]=\varnothing\right]=\mathbf{P}[\mathcal{L}<\infty]=\exp \left(-\frac{1}{3} L+O(1)\right) \quad \text { as } L \rightarrow \infty
$$

\subsection{Generalized Cardy's formula}

By conformal invariance, we may work in the half-plane H. Map the rectangle $\mathcal{R}$ conformally onto $\mathbf{H}$ so that $A_{1}$ is mapped to $1, A_{2}$ is mapped to $\infty, A_{3}$ is mapped to 0 , and then the image $x=x(L) \in(0,1)$ of $A_{4}$ is determined for us. Let $K_{t}$ be the hull of an SLE $_{6}$-process $g_{t}=g_{K_{t}}$ in $\mathbf{H}$, with driving process $W(t)$, which is started at $W(0)=x$ (that is, $K_{t}$ is a translation by $x$ of the standard SLE $_{6}$ starting at 0 ). In order to emphasize the dependence on $x$, we will use the notation $\mathbf{P}_{x}$ and $\mathbf{E}_{x}$ for probability and expectation.

Set

$$
\begin{aligned}
T_{0} & :=\sup \left\{t \geqslant 0: \bar{K}_{t} \cap(-\infty, 0]=\varnothing\right\}, \\
T_{1} & :=\sup \left\{t \geqslant 0: \bar{K}_{t} \cap[1, \infty)=\varnothing\right\}, \\
T & :=\min \left\{T_{0}, T_{1}\right\} .
\end{aligned}
$$


As will be demonstrated, $T_{0}, T_{1}<\infty$ a.s. Let

$$
f_{t}(z):=\frac{g_{t}(z)-g_{t}(0)}{g_{t}(1)-g_{t}(0)}
$$

for $t<T$, which is just $g_{t}$ renormalized to fix 0,1 and $\infty$. It turns out that $f_{T}:=\lim _{t \nearrow T} f_{t}$ exists a.s. On the event $T_{1}>T_{0}, f_{T}$ uniformizes the quadrilateral

$$
\left(\mathbf{H} \backslash K_{T} ; 1, \infty, \min \left(\bar{K}_{T} \cap \mathbf{R}\right), \max \left(\bar{K}_{T} \cap \mathbf{R}\right)\right)
$$

to the form

$$
\left(\mathbf{H} ; 1, \infty, 0, f_{T}\left(\max \left(\bar{K}_{T} \cap \mathbf{R}\right)\right)\right)
$$

Therefore, we want to know the distribution of

$$
1-f_{T}\left(\max \left(\bar{K}_{T} \cap \mathbf{R}\right)\right)
$$

and how it depends on $x$ (especially when $x \in(0,1)$ is close to 1 ). In this subsection, we will calculate something very closely related: the distribution of $f_{T}^{\prime}(1)$ and how it depends on $x$.

Set

$$
\Lambda(1-x, b):=\mathbf{E}_{x}\left[1_{\left\{T_{0}<T_{1}\right\}} f_{T}^{\prime}(1)^{b}\right]
$$

for $b \geqslant 0$ and $x \in(0,1)$. Recall the definition of the hypergeometric function ${ }_{2} F_{1}$ (see, e.g., $[30])$ :

$$
{ }_{2} F_{1}\left(a_{0}, a_{1}, a_{2} ; x\right)=\sum_{n=0}^{\infty} \frac{\left(a_{0}\right)_{n}\left(a_{1}\right)_{n}}{\left(a_{2}\right)_{n} n !} x^{n},
$$

where $(a)_{n}=\prod_{j=1}^{n}(a+j-1)$ and $(a)_{0}=1$. Note that ${ }_{2} F_{1}\left(a_{0}, a_{1}, a_{2} ; 0\right)=1$.

THEOREM 3.2. For all $b \geqslant 0, x \in(0,1)$,

$$
\Lambda(x, b)=\frac{\sqrt{\pi} 2^{-2 \hat{b}} \Gamma\left(\frac{5}{6}+\hat{b}\right)}{\Gamma\left(\frac{1}{3}\right) \Gamma(1+\hat{b})} x_{2}^{1 / 6+\hat{b}} F_{1}\left(\frac{1}{6}+\hat{b}, \frac{1}{2}+\hat{b}, 1+2 \hat{b} ; x\right)
$$

where

$$
\hat{b}=\frac{1}{6} \sqrt{1+24 b}
$$

and ${ }_{2} F_{1}$ is the hypergeometric function.

Setting $b=0$, we obtain Cardy's formula [7]. Thus, this result can be thought of as a generalization of Cardy's formula.

Note that Theorem 3.2 determines completely the law of $1_{\left\{T_{0}<T_{1}\right\}} f_{T}^{\prime}(1)$. In particular, the Laplace transform of the conditional law of $\log 1 / f_{T}^{\prime}(1)$ given $\left\{T_{0}<T_{1}\right\}$ is $\Lambda(1-x, b) / \Lambda(1-x, 0)$. 
Proof. We first observe that $T_{1}<\infty$ almost surely. Indeed, $g_{t}(1)-W(t)$ is a Bessel process with index $\frac{5}{3}$, with time linearly scaled, and hence hits 0 almost surely in finite time (e.g. [39]). Similarly, $g_{t}(0)-W(t)$ hits 0 almost surely. It is clear that $T_{1}$ is the time $t$ when $g_{t}(1)-W(t)$ hits 0 , and $T_{0}$ is the time when $g_{t}(0)-W(t)$ hits 0 . It follows from Theorem 2.6 and $x \in(0,1)$ that almost surely $T_{0} \neq T_{1}$. We may also conclude that

$$
\lim _{x \rightarrow 0} \mathbf{P}_{x}\left[T_{0}<T_{1}\right]=1, \quad \lim _{x \rightarrow 1} \mathbf{P}_{x}\left[T_{0}<T_{1}\right]=0
$$

The next goal is to prove that

$$
\lim _{t \nearrow T} f_{t}^{\prime}(1)>0 \quad \text { if and only if } \quad T_{0}<T_{1}
$$

If $T_{0}<T_{1}$, then $\bar{K}_{T} \cap[1, \infty]=\varnothing$. Therefore $f_{T}$ is defined and conformal near 1 , and $f_{T}^{\prime}(1)>0$, by the reflection principle. On the other hand, if $T_{1}<T_{0}$, then $\bar{K}_{T} \cap[1, \infty) \neq \varnothing$. We claim that

$$
1 \notin \bar{K}_{T_{1}} \quad \text { almost surely; }
$$

since $\bar{K}_{T_{1}} \cap[1, \infty) \neq \varnothing$, this means that $K_{T_{1}}$ separates 1 from $\infty$ in $\mathbf{H}$. Indeed, let $\phi: \mathbf{H} \rightarrow \mathbf{H}$ be the anti-conformal automorphism that fixes $x$ and exchanges 1 and $\infty . T_{1}<\infty$ a.s. and $\sup _{t<T_{1}}|W(t)|<\infty$ a.s. imply that $K_{T_{1}}$ is bounded a.s., which is the same as saying that $\phi\left(K_{t}\right)$ stays bounded away from 1 as $t \nearrow T_{1}$. But Corollary 2.3 and invariance under reflection imply that up to time $T_{1}$ the law of $K_{t}$ is the same as a time change of the law of $\phi\left(K_{t}\right)$. Hence, a.s. $K_{t}$ stays bounded away from 1 as $t \nearrow T_{1}$, proving (3.7). It follows from (3.7) that $\lim _{t>T_{1}} f_{t}^{\prime}(1)=0$ a.s. on the event $T_{1}<T_{0}$ (observe that, given (3.7), the extremal length from a neighborhood of 0 to a neighborhood of 1 in $\mathbf{H} \backslash K_{t}$ tends to $\infty$ as $t \nearrow T_{1}$ ), and (3.6) is established.

Define the renormalized version of $W(t)$,

$$
Z(t):=\frac{W(t)-g_{t}(0)}{g_{t}(1)-g_{t}(0)}
$$

and the new time parameter

$$
s=s(t):=\int_{0}^{t} \frac{d t}{\left(g_{t}(1)-g_{t}(0)\right)^{2}}, \quad t<T .
$$

Set $s_{0}:=\lim _{t \nearrow T} s(t)$. Since $T_{1} \neq T_{0}$ a.s., $\inf \left\{g_{t}(1)-g_{t}(0): t<T\right\}>0$ a.s., and hence $s_{0}<\infty$ a.s. Let $t(s)$ denote the inverse to the map $t \mapsto s(t)$. A direct calculation gives

$$
\partial_{s}\left(f_{t(s)}(z)\right)=\frac{-2}{Z(t)-f_{t}(z)}+\frac{2\left(1-f_{t}(z)\right)}{Z(t)}-\frac{2 f_{t}(z)}{1-Z(t)}
$$


and

$$
d Z(t)=\frac{d W_{t}}{g_{t}(1)-g_{t}(0)}+\frac{2 d t}{\left(g_{t}(1)-g_{t}(0)\right)^{2}}\left(\frac{1}{Z(t)}+\frac{1}{Z(t)-1}\right) .
$$

We now use the notation

$$
\widetilde{Z}(s):=Z(t(s)), \quad \tilde{f}_{s}(z):=f_{t(s)}(z)
$$

Then,

$$
d \widetilde{Z}_{s}=d X_{s}+\frac{2(1-2 \widetilde{Z}(s)) d s}{\widetilde{Z}(s)(1-\widetilde{Z}(s))}=d X_{s}+\left(\frac{2}{\widetilde{Z}(s)}-\frac{2}{1-\widetilde{Z}(s)}\right) d s
$$

where $\left(X_{s}, s \geqslant 0\right)$ has the same law as $(W(t), t \geqslant 0)$; i.e., it is a Brownian motion with time rescaled by a factor of 6 . Also,

$$
\partial_{s}\left(\tilde{f}_{s}(z)\right)=\frac{-2}{\widetilde{Z}(s)-\tilde{f}_{s}(z)}+\frac{2\left(1-\tilde{f}_{s}(z)\right)}{\widetilde{Z}(s)}-\frac{2 \tilde{f}_{s}(z)}{1-\widetilde{Z}(s)}
$$

These two equations describe the evolution of $\tilde{f}_{s}(z)$. Note that $s(T)=s_{0}$ is the first time at which $\widetilde{Z}(s)$ hits 0 or 1 .

We now assume that $b>0$. Differentiating (3.9) with respect to $z$ gives

$$
\partial_{s}\left(\log \tilde{f}_{s}^{\prime}(z)\right)=\frac{-2}{\left(\widetilde{Z}(s)-\tilde{f}_{s}(z)\right)^{2}}-\frac{2}{\widetilde{Z}(s)}-\frac{2}{1-\widetilde{Z}(s)}
$$

(the Cauchy integral formula, for example, shows that we may indeed differentiate, but this is also legitimate since $\partial_{s}$ and $\partial_{z}$ commute in this case). We are particularly interested in

$$
\alpha(s):=\log \tilde{f}_{s}^{\prime}(1)=\log f_{t(s)}^{\prime}(1)
$$

which satisfies

$$
\partial_{s} \alpha(s)=\frac{-2}{(\widetilde{Z}(s)-1)^{2}}-\frac{2}{\widetilde{Z}(s)}-\frac{2}{1-\widetilde{Z}(s)} .
$$

Note that the equations (3.8) and (3.11) describe the evolution of the Markov process $(\widetilde{Z}(s), \alpha(s))$. The process stops at $s_{0}$. Define

$$
y(x, v):=\mathbf{E}\left[\exp \left(b \alpha\left(s_{0}\right)\right) \mid \widetilde{Z}(0)=x, \alpha(0)=v\right]
$$

where the expectation corresponds to the Markov process started from $\widetilde{Z}(0)=x$ and $\alpha(0)=v$. From the definition of $y$ it follows that

$$
y(x, 0)=\Lambda(1-x, b)
$$


since $\lim _{t \nearrow T} f_{t}=f_{T}$ in a neighborhood of 1 on the event $T_{0}<T_{1}$ and (3.6) holds. It is standard that such a function $y(x, v)$ is $C^{\infty}$, and the strong Markov property ensures that the process

$$
Y=y(\widetilde{Z}(s), \alpha(s))
$$

is a local martingale. The drift term in Itô's formula for $d Y$ must vanish, which gives

$$
0=\frac{2(1-2 x)}{x(1-x)} \partial_{x} y+3 \partial_{x x}^{2} y+\left(\frac{-2}{(1-x)^{2}}-\frac{2}{x}-\frac{2}{1-x}\right) \partial_{v} y .
$$

As

$$
\alpha(s)=\alpha(0)+\int_{0}^{u}\left(\partial_{s} \alpha\left(s^{\prime}\right)\right) d s^{\prime},
$$

we get

$$
y(x, v)=\exp (b v) y(x, 0) .
$$

Set

$$
h(x):=y(1-x, 0)=\Lambda(x, b),
$$

so that $y(x, v)=\exp (b v) h(1-x)$. Hence $(3.12)$ becomes

$$
-2 b h(x)+2 x(1-2 x) h^{\prime}(x)+3 x^{2}(1-x) h^{\prime \prime}(x)=0 .
$$

The second statement in (3.5) implies that

$$
\lim _{x \searrow 0} h(x)=0
$$

while

$$
\lim _{x>1} h(x)=1
$$

holds, since when $x$ is close to $0, K_{T_{0}}$ is likely to be small, by scale invariance, for example. The differential equation (3.13) can be solved explicitly by looking for solutions of the type $h(x)=x^{c} z(x)$ : two linearly independent solutions are $(i=1,2)$

$$
h_{i}(x)=x^{1 / 6+b_{i}}{ }_{2} F_{1}\left(\frac{1}{6}+b_{i}, \frac{1}{2}+b_{i}, 1+2 b_{i} ; x\right),
$$

where

$$
b_{1}=-b_{2}=\frac{1}{6} \sqrt{1+24 b} .
$$

Recall that ${ }_{2} F_{1}\left(a_{0}, a_{1}, a_{2} ; 0\right)=1$. The function $h(x)$ must be a linear combination of $h_{1}$ and $h_{2}$. However, $\lim _{x \searrow^{\prime} 0} h(x)=0=\lim _{x \succ 0} h_{1}(x)$, but $\lim _{x \backslash 0} h_{2}(x)=\infty$. Hence, $h(x)=$ $c h_{1}(x)$ for some constant $c$. The equality $h(1)=1$ and knowledge of the value at $x=1$ of 
hypergeometric functions (see, e.g., [30]) allows the determination of $c$, and establishes the theorem in the case $b>0$. The case $b=0$ follows by taking a limit as $b \searrow 0$.

Remark. With the same proof, Theorem 3.2 generalizes to $\mathrm{SLE}_{\kappa}$ with $\kappa>4$, and gives

$$
\Lambda_{\kappa}(x, b)=C(b, \kappa) x^{1 / 2-2 / \kappa+\hat{b}_{\kappa}}{ }_{2} F_{1}\left(\frac{1}{2}-2 / \kappa+\hat{b}_{\kappa}, 6 / \kappa-\frac{1}{2}+\hat{b}_{\kappa}, 1+2 \hat{b}_{\kappa} ; x\right)
$$

where

$$
\hat{b}_{\kappa}:=\frac{\sqrt{(\kappa-4)^{2}+16 \kappa b}}{2 \kappa}, \quad C(b, \kappa):=\frac{\Gamma\left(\frac{3}{2}-6 / \kappa+\hat{b}_{\kappa}\right) \Gamma\left(\frac{1}{2}+2 / \kappa+\hat{b}_{\kappa}\right)}{\Gamma(1-4 / \kappa) \Gamma\left(1+2 \hat{b}_{\kappa}\right)} .
$$

\subsection{Determination of the SLE -exponents $^{-}$}

For every $t \geqslant 0$, set

$$
M_{t}:=\max \left(\bar{K}_{t} \cap \mathbf{R}\right)
$$

The following lemma shows that our understanding of the derivative $f_{T}^{\prime}(1)$ gives information on $f_{T}\left(M_{T}\right)$ itself.

LEMMA 3.3. In the above setting, let $N_{T}:=f_{T}\left(M_{T}\right)$. For $b \geqslant 0$, set

$$
\Theta(x, b):=E_{1-x}\left[1_{\left\{T_{0}<T_{1}\right\}}\left(1-N_{T}\right)^{b}\right] .
$$

Then

$$
\left(\frac{1}{2} x\right)^{b} \Lambda\left(\frac{1}{2} x, b\right) \leqslant \Theta(x, b) \leqslant x^{b} \Lambda(x, b) .
$$

Note that $\Theta(x, b)$ is close to the quantity we are after, since $-\log \left(1-N_{T}\right)$ is approximately the extremal length of the quadrilateral

$$
\left(\mathbf{H} \backslash K_{T} ; \min \left(\bar{K}_{T} \cap \mathbf{R}\right), \max \left(\bar{K}_{T} \cap \mathbf{R}\right), 1, \infty\right) .
$$

Proof. It follows easily from (3.10) that $f_{t}^{\prime}(z)$ is non-decreasing in $z$ (viewed as a real variable), as long as $z \geqslant M_{t}$. Therefore,

$$
1-N_{T}=\int_{M_{T}}^{1} f_{T}^{\prime}(z) d z \leqslant\left(1-M_{T}\right) f_{T}^{\prime}(1) \leqslant x f_{T}^{\prime}(1) .
$$

This gives the right-hand inequality in (3.14).

To get the other inequality, consider some fixed $x^{*}>1\left(x^{*}\right.$ should be thought of as close to 1 ; we will eventually take $\left.x^{*}=1 /(1-x)\right)$. Let $\tilde{x}^{*}=f_{T}\left(x^{*}\right)$. Then

$$
\tilde{x}^{*}-N_{T} \geqslant \tilde{x}^{*}-1=\int_{1}^{x^{*}} f_{T}^{\prime}(z) d z \geqslant\left(x^{*}-1\right) f_{T}^{\prime}(1) .
$$


This gives

$$
E_{1-x}\left[1_{\left\{T_{0}<T_{1}\right\}}\left(\tilde{x}^{*}-N_{T}\right)^{b}\right] \geqslant\left(x^{*}-1\right)^{b} \Lambda(x, b)
$$

A simple scaling argument will give an upper bound of the left-hand side of this inequality in terms of $\Theta$. Let

$$
T^{*}=\inf \left\{t \geqslant 0: \bar{K}_{t} \cap \mathbf{R} \backslash\left(0, x^{*}\right) \neq \varnothing\right\}
$$

Note that $T \leqslant T^{*}<\infty$ a.s. and that $T=T^{*}$ if $T_{0}<T_{1}$. For each $t \leqslant T^{*}$, let $f_{t}^{*}$ be the conformal map from the unbounded component of $\mathbf{H} \backslash K_{t}$ to $\mathbf{H}$, which fixes the points $\infty, 0, x^{*}$. For all $t \leqslant T$,

$$
f_{t}^{*}(z)=\frac{x^{*}}{f_{t}\left(x^{*}\right)} f_{t}(z)
$$

Note that

$$
f_{t}\left(x^{*}\right) \leqslant x^{*}=f_{t}^{*}\left(x^{*}\right)
$$

Then,

$$
1_{\left\{T_{0}<T_{1}\right\}}\left(\tilde{x}^{*}-N_{T}\right) \leqslant 1_{\left\{T_{0}<T_{1}\right\}}\left(x^{*}-f_{T}^{*}\left(M_{T}\right)\right)
$$

Hence,

$$
1_{\left\{T_{0}<T_{1}\right\}}\left(\tilde{x}^{*}-N_{T}\right) \leqslant 1_{\left\{M_{\left.T^{*}<x^{*}\right\}}\right.}\left(x^{*}-f_{T^{*}}^{*}\left(M_{T^{*}}\right)\right),
$$

since on the event $T_{0}<T_{1}$, we have $T=T^{*}$ and $M_{T}=M_{T^{*}}$. However, by scale invariance, when $W(0)=1-x$ the random variable

$$
1_{\left\{M_{\left.T^{*}<x^{*}\right\}}\right.}\left(x^{*}-f_{T^{*}}^{*}\left(M_{T^{*}}\right)\right)
$$

has the same law as the random variable

$$
x^{*} 1_{\left\{M_{T}<1\right\}}\left(1-f_{T}\left(M_{T}\right)\right)
$$

does when $W(0)=(1-x) / x^{*}$. Thus, combining (3.16) and (3.15) gives

$$
\left(x^{*}\right)^{b} \Theta\left(1-(1-x) / x^{*}, b\right) \geqslant\left(x^{*}-1\right)^{b} \Lambda(x, b) .
$$

We take $x^{*}=1 /(1-x)$, say, and get

$$
\Theta(2 x, b) \geqslant \Theta\left(2 x-x^{2}, b\right) \geqslant x^{b} \Lambda(x, b)
$$

which gives the left-hand side of (3.14).

Proof of Theorem 3.1. We are now ready to derive Theorem 3.1 by combining Theorem 3.2 and Lemma 3.3. 
In the setting of the theorem, let $\phi: \mathcal{R}(L) \rightarrow \mathbf{H}$ be the conformal homeomorphism satisfying $\phi\left(A_{1}\right)=1, \phi\left(A_{2}\right)=\infty$ and $\phi\left(A_{3}\right)=0$. Set $x=x(L):=\phi\left(A_{4}\right)$. By conformal invariance, the law of $\mathcal{L}$ is the same as that of the $\pi$-extremal distance $\mathcal{L}^{*}$ from $(-\infty, 0]$ to $\left(N_{T}, 1\right)$ in $\mathbf{H}$ (with the notations of Lemma 3.3). Considering the map $z \mapsto \log (z-1)$ makes it clear that

$$
L=-\log (1-x)+O(1)
$$

for $L>1$, and similarly

$$
\mathcal{L}^{*}=-\log \left(1-N_{T}\right)+O(1) .
$$

For $L>1$ (note also that $\mathcal{L} \geqslant L$ ),

$$
\begin{aligned}
\mathbf{E}\left[1_{\{\mathcal{L}<\infty\}} \exp (-\lambda \mathcal{L})\right] & =\mathbf{E}\left[1_{\left\{\mathcal{L}^{*}<\infty\right\}} \exp \left(-\lambda \mathcal{L}^{*}\right)\right] \\
& =\exp (O(1)) \mathbf{E}\left[1_{\left\{N_{T}<1\right\}}\left(1-N_{T}\right)^{\lambda}\right] \quad(\text { by }(3.18)) \\
& =\exp (O(1)) \Theta(1-x, \lambda) \\
& =\exp (O(\lambda+1))(1-x)^{u(\lambda)} \quad \text { (by Lemma } 3.3 \text { and Theorem 3.2) } \\
& =\exp (O(\lambda+1)) \exp (-u(\lambda) L) \quad(\text { by }(3.17))
\end{aligned}
$$

which completes the proof of Theorem 3.1.

\section{The Brownian half-plane exponents}

We are now ready to combine the results collected so far and a "universality" idea similar to that developed in [29] to compute the exact value of some Brownian intersection exponents in the half-plane.

\subsection{Definitions and background}

In this short subsection, we quickly review some results on intersection exponents between independent planar Brownian motions. For details and complete proofs of these results, see [28], [29] .

Suppose that $n+p$ independent planar Brownian motions $\beta^{1}, \ldots, \beta^{n}$ and $\gamma^{1}, \ldots, \gamma^{p}$ are started from points $\beta^{1}(0)=\ldots=\beta^{n}(0)=0$ and $\gamma^{1}(0)=\ldots=\gamma^{p}(0)=1$ in the complex plane, and consider the probability $f_{n, p}(t)$ that for all $j \leqslant n$ and $l \leqslant p$, the paths of $\beta^{j}$ up to time $t$ and of $\gamma^{l}$ up to time $t$ do not intersect; more precisely,

$$
f_{n, p}(t):=\mathbf{P}\left[\left(\bigcup_{j=1}^{n} \beta^{j}[0, t]\right) \cap\left(\bigcup_{l=1}^{p} \gamma^{l}[0, t]\right)=\varnothing\right] .
$$


It is easy to see that as $t \rightarrow \infty$ this probability decays roughly like a power of $t$. The $(n, p)$-intersection exponent $\xi(n, p)$ is defined as twice this power, i.e.,

$$
f_{n, p}(t)=(\sqrt{t})^{-\xi(n, p)+o(1)}, \quad t \rightarrow \infty .
$$

We call $\xi(n, p)$ the intersection exponent between one packet of $n$ Brownian motions and one packet of $p$ Brownian motions (for a list of references on Brownian intersection exponents, see [28]). Note that the exponent $\zeta$ described in the introduction is $\frac{1}{2} \xi(1,1)$. It turns out to be more convenient to use this definition as a power of $\sqrt{t}$, i.e., of the space parameter. A Brownian motion travels very roughly to distance $\sqrt{t}$ in time $t$ : recall that if $\beta$ is a planar Brownian motion started from 0 , say, and $T_{R}$ denotes its hitting time of the circle of radius $R$ about 0 , then for all $\delta>0$, the probability that $T_{R} \notin\left(R^{2-\delta}, R^{2+\delta}\right)$ decays as $R \rightarrow \infty$ faster than any negative power of $R$. This facilitates an easy conversion between the time-based definition of intersection exponents and a definition where the particles die when they exit a large ball.

Similarly, one can define corresponding probabilities for intersection exponents in a half-plane,

$$
\tilde{f}_{n, p}(t):=\mathbf{P}\left[\forall j \leqslant n, \forall l \leqslant p, \beta^{j}[0, t] \cap \gamma^{l}[0, t]=\varnothing \text { and } \beta^{j}[0, t] \cup \gamma^{l}[0, t] \subset \mathcal{H}\right]
$$

where $\mathcal{H}$ is some half-plane containing the two starting points $\left(\tilde{f}_{n, p}(t)\right.$ will depend on $\left.\mathcal{H}\right)$. In plain words, we are looking at the probability that all Brownian motions stay in the half-plane and that all $\beta$ 's avoid all $\gamma$ 's. It is also easy to see that there exists a $\tilde{\xi}(n, p)$ (which does not depend on $\mathcal{H}$ ) such that

$$
\tilde{f}_{n, p}(t)=(\sqrt{t})^{-\tilde{\xi}(n, p)+o(1)}, \quad t \rightarrow \infty .
$$

Note that $\tilde{\zeta}$ described in the introduction is $\frac{1}{2} \tilde{\xi}(1,1)$.

One can also define intersection exponents $\xi\left(n_{1}, \ldots, n_{p}\right)$ and $\tilde{\xi}\left(n_{1}, \ldots, n_{p}\right)$ involving more packets of Brownian motions. (For a more detailed discussion of this, see [28]). For instance, if $B^{1}, B^{2}, B^{3}, B^{4}$ denote four Brownian motions started from different points, the exponent $\xi(2,1,1)$ is defined by

$$
\begin{aligned}
\mathbf{P}\left[\text { the three sets } B^{1}[0, t] \cup B^{2}[0, t], B^{3}[0, t], B^{4}[0, t] \text { are disjoint }\right] & \\
& =t^{-\xi(2,1,1) / 2+o(1)}, \quad t \rightarrow \infty .
\end{aligned}
$$

One of the results of [28] is that there is a natural and rigorous way to generalize the definition of intersection exponents between packets of Brownian motions to the case where each packet of Brownian motions is the union of a "non-integer number" of paths; for 
the half-plane exponents, one can define the exponents $\tilde{\xi}\left(u_{1}, \ldots, u_{p}\right)$, where $u_{1}, \ldots, u_{p} \geqslant 0$. These generalized exponents satisfy the so-called cascade relations (see [28]): for any $1 \leqslant q \leqslant p-1$,

$$
\tilde{\xi}\left(u_{0}, \ldots, u_{p}\right)=\tilde{\xi}\left(u_{0}, \ldots, u_{q-1}, \tilde{\xi}\left(u_{q}, \ldots, u_{p}\right)\right) .
$$

Moreover, $\tilde{\xi}$ is invariant under a permutation of its arguments.

There exists (see [28], [29]) a characterization of these exponents in terms of the so-called Brownian excursions that turns out to be useful. For any bounded simplyconnected open domain $D$, there exists a Brownian excursion measure $\mu_{D}$ in $D$. This is an infinite measure on paths $(B(t), t \leqslant \tau)$ in $D$ such that $B(0, \tau) \subset D$ and $B(0), B(\tau) \in \partial D$ (these can be viewed as prime ends if necessary). $x_{s}:=B(0)$ and $x_{e}:=B(\tau)$ are the starting point and terminal point of the excursion. One possible definition of $\mu_{D}$ is the following: Suppose first that $D$ is the unit disc. For any $s>0$ define the measure $P^{s}$ on Brownian paths (modulo continuous increasing time change) started uniformly on the circle of radius $\exp (-s)$, and killed when they exit $D$. Note for any $s_{0}>s$, the killed Brownian path defined under the probability measure $P^{s}$ has a probability $s / s_{0}$ to intersect the circle of radius $\exp \left(-s_{0}\right)$. Then, define

$$
\mu_{D}:=\lim _{s \searrow 0}(2 \pi / s) P^{s}
$$

One can then easily check that for any Möbius transformation $\phi$ from $D$ onto $D$, $\phi\left(\mu_{D}\right)=\mu_{D}$. This makes it possible to extend the definition of $\mu_{D}$ to any simplyconnected domain $D$, by conformal invariance. These Brownian excursions also have a "restriction" property [29], as the Brownian paths only feel the boundary of $D$ when they hit it (and get killed).

Suppose for a moment that $\mathcal{R}=\mathcal{R}(L) \subset \mathbf{C}$ is the rectangle with corners given by (3.1), and that $\mathcal{B}$ is the trace of the Brownian excursion $(B(t), t \leqslant \tau)$ in $\mathcal{R}$. Define the event

$$
E_{1}=\left\{B(0) \in\left[A_{1}, A_{4}\right] \text { and } B(\tau) \in\left[A_{2}, A_{3}\right]\right\},
$$

i.e., $B$ crosses the rectangle from the left to the right. (Although $\mu_{\mathcal{R}}$ is an infinite measure, $\mu_{\mathcal{R}}\left(E_{1}\right)$ is finite.) When $E_{1}$ holds, let $\mathcal{R}_{B}^{+}$be the component of $\mathcal{R} \backslash \mathcal{B}$ above $\mathcal{B}$, and let $\mathcal{R}_{B}^{-}$be the component of $\mathcal{R} \backslash \mathcal{B}$ below $\mathcal{B}$. Let $\mathcal{L}_{B}^{-}$(resp. $\mathcal{L}_{B}^{+}$) denote the $\pi$-extremal distance between $\left[A_{1}, x_{s}\right]$ and $\left[A_{2}, x_{e}\right]$ in $\mathcal{R}_{B}^{-}$(resp. $\left[x_{s}, A_{4}\right]$ and $\left[x_{e}, A_{3}\right]$ ) in $\mathcal{R}_{B}^{+}$.

Then, for any $\alpha \geqslant 0$ and $\alpha^{\prime} \geqslant 0$, the exponent $\tilde{\xi}\left(\alpha, 1, \alpha^{\prime}\right)=\tilde{\xi}\left(1, \tilde{\xi}\left(\alpha, \alpha^{\prime}\right)\right)$ is characterized by

$$
\mathbf{E}_{\mu_{\mathcal{R}}}\left[1_{E_{1}} \exp \left(-\alpha \mathcal{L}_{B}^{+}-\alpha^{\prime} \mathcal{L}_{B}^{-}\right)\right]=\exp \left(-\tilde{\xi}\left(\alpha^{\prime}, 1, \alpha\right) L+o(L)\right)
$$

when $L \rightarrow \infty$, where $\mathbf{E}_{\mu_{\mathcal{R}}}$ denotes expectation (that is, integration) with respect to the measure $\mu_{\mathcal{R}}$. Similarly,

$$
\mathbf{E}_{\mu_{\mathcal{R}}}\left[1_{E_{1}} \exp \left(-\alpha \mathcal{L}_{B}^{+}\right)\right]=\exp (-\tilde{\xi}(1, \alpha) L+o(L)), \quad L \rightarrow \infty
$$


See [29]. It will also be important later that $\tilde{\xi}$ is continuous in its arguments, and that $\lambda \mapsto \tilde{\xi}(1, \lambda)$ is strictly monotone.

\subsection{Statement and proof}

For any $p \geqslant 0$, we put

$$
v_{p}=\frac{1}{6} p(p+1) .
$$

Let $\mathcal{V}$ denote the set of numbers $\left\{v_{p}: p \in \mathbf{N}\right\}$. Note that the smallest values in $\mathcal{V}$ are $0, \frac{1}{3}, 1,2, \frac{10}{3}, 5,7$.

We are now ready to prove the following result:

TheOREM 4.1. For any $k \geqslant 2, \alpha_{1}, \ldots, \alpha_{k-1}$ in $\mathcal{V}$, and for all $\alpha_{k} \in \mathbf{R}_{+}$,

$$
\tilde{\xi}\left(\alpha_{1}, \ldots, \alpha_{k}\right)=\frac{1}{24}\left(\left(\sqrt{24 \alpha_{1}+1}+\ldots+\sqrt{24 \alpha_{k}+1}-(k-1)\right)^{2}-1\right)
$$

It is immediate to verify that this theorem implies Theorem 1.1.

Remark. In [26], Theorem 4.1 is extended to all non-negative reals $\alpha_{1}, \ldots, \alpha_{k}$.

Theorem 4.1 is a consequence of the cascade relations and the following lemma, which is the special case of the theorem with $k=2, \alpha_{1}=\frac{1}{3}$ :

Lemma 4.2. For any $\lambda>0$,

$$
\tilde{\xi}\left(\frac{1}{3}, \lambda\right)=u(\lambda)
$$

where $u(\lambda)$ is given by (3.2).

Proof of Theorem 4.1 (assuming Lemma 4.2). Define $U(\lambda)=\sqrt{24 \lambda+1}-1$, for all $\lambda \geqslant 0$. Lemma 4.2 implies immediately that for all $\lambda \geqslant 0$,

$$
U\left(\tilde{\xi}\left(\frac{1}{3}, \lambda\right)\right)=U(\lambda)+2=U(\lambda)+U\left(\frac{1}{3}\right)
$$

and (for all integer $p) v_{p+1}=\tilde{\xi}\left(\frac{1}{3}, v_{p}\right)$. The cascade relations then imply that for all integers $p_{1}, \ldots, p_{k-1}$

$$
\tilde{\xi}\left(v_{p_{1}}, \ldots, v_{p_{k-1}}, \lambda\right)=U^{-1}\left(2\left(p_{1}+\ldots+p_{k-1}\right)+U(\lambda)\right) .
$$

This is (4.4).

Proof of Lemma 4.2. For convenience, we again work in a rectangle rather than in the upper half-plane. Let $\mathcal{R}=\mathcal{R}(L)$, and let $\mathcal{S}$ denote the closure of the hull of $\mathrm{SLE}_{6}$ from $A_{4}$ to $\left[A_{1}, A_{2}\right] \cup\left[A_{2}, A_{3}\right]$ in $\mathcal{R}$, as in Theorem 3.1. Let $\mathcal{B}$ denote the trace of a Brownian 
excursion in $\mathcal{R}$; we will call its starting point $x_{s}$ and its terminal point $x_{e}$. Consider the events

$$
\begin{aligned}
& E_{1}=\left\{x_{s} \in\left[A_{1}, A_{4}\right] \text { and } x_{e} \in\left[A_{2}, A_{3}\right]\right\}, \\
& E_{2}=\left\{\mathcal{S} \cap\left[A_{1}, A_{2}\right]=\varnothing\right\}, \\
& E_{3}=E_{1} \cap E_{2} \cap\{\mathcal{S} \cap \mathcal{B}=\varnothing\} .
\end{aligned}
$$

When $E_{2}$ holds, let $\mathcal{L}_{S}$ denote the $\pi$-extremal distance between the vertical edges of $\mathcal{R}$ in $\mathcal{R} \backslash \mathcal{S}$ (that is, in the quadrilateral "below" $\mathcal{S}$ ). Otherwise, let $\mathcal{L}_{S}=\infty$.

When $E_{1}$ holds, let $\mathcal{R}_{B}^{+}$be the component of $\mathcal{R} \backslash \mathcal{B}$ above $\mathcal{B}$, and let $\mathcal{R}_{B}^{-}$be the component of $\mathcal{R} \backslash \mathcal{B}$ below $\mathcal{B}$. Let $\mathcal{L}_{B}^{-}$(resp. $\mathcal{L}_{B}^{+}$) denote the $\pi$-extremal distance between the vertical edges of $\mathcal{R}$ in $\mathcal{R}_{B}^{-}$(resp. in $\mathcal{R}_{B}^{+}$), as before. When $E_{3}$ holds, let $\mathcal{L}_{S B}$ denote the $\pi$-extremal distance between the vertical edges of $\mathcal{R}$ in $\mathcal{R}_{B}^{+} \backslash \mathcal{S}$ (that is, in the quadrilateral "below $\mathcal{S}$ and above $\mathcal{B}$ ").

Let $\lambda>0$. We are interested in the asymptotic behavior of

$$
f(L)=\mathbf{E}\left[1_{E_{3}} \exp \left(-\lambda \mathcal{L}_{S B}\right)\right]
$$

when $L \rightarrow \infty$. By first taking expectations with respect to $B$ (with the measure $\mu_{\mathcal{R}}$ ), and using the restriction property (Corollary 2.4) for the domains $\mathcal{R}$ and $\mathcal{R}_{B}^{+}$, it follows that as $L \rightarrow \infty$,

$$
\begin{aligned}
f(L) & =\mathbf{E}_{B}\left[\mathbf{E}_{S}\left[\exp \left(-\lambda \mathcal{L}_{S B}\right)\right]\right] & & \\
& =\mathbf{E}_{B}\left[\exp \left(-u(\lambda) \mathcal{L}_{B}^{+}+O(1)\right)\right] & & \text { (by Theorem 3.1 and restriction to } \left.\mathcal{R}_{B}^{+}\right) \\
& =\exp (-\tilde{\xi}(1, u(\lambda)) L+o(L)) & & (\text { by }(4.3)) .
\end{aligned}
$$

On the other hand, we may first take expectation with respect to $\mathcal{S}$. Given $\mathcal{S}$, the law of $\mathcal{L}_{S B}$ is the same as that of $\mathcal{L}_{B}^{-}$, by complete conformal invariance of the excursion measure (which is the analogue of the restriction property to the excursion measure, see [28]). Hence, as $L \rightarrow \infty$,

$$
\begin{array}{rlr}
f(L) & =\mathbf{E}_{S}\left[\mathbf{E}_{B}\left[1_{E_{3}} \exp \left(-\lambda \mathcal{L}_{S B}\right)\right]\right] & \\
& =\mathbf{E}_{S}\left[\mathbf{E}_{B}\left[1_{E_{3}} \exp \left(-\lambda \mathcal{L}_{B}^{-}\right)\right]\right] & \\
& =\mathbf{E}_{B}\left[\mathbf{E}_{S}\left[1_{E_{3}} \exp \left(-\lambda \mathcal{L}_{B}^{-}\right)\right]\right] \\
& =\mathbf{E}_{B}\left[\mathbf{P}_{S}\left[E_{3} \mid \mathcal{L}_{B}^{+}\right] \exp \left(-\lambda \mathcal{L}_{B}^{-}\right)\right] & \\
& =\mathbf{E}_{B}\left[\exp \left(-\frac{1}{3} \mathcal{L}_{B}^{+}+O(1)\right) \exp \left(-\lambda \mathcal{L}_{B}^{-}\right)\right] & \text {(by }(3.4)) \\
& =\exp \left(-\tilde{\xi}\left(\frac{1}{3}, 1, \lambda\right) L+o(L)\right) & \text { (by (4.2)) } \\
& =\exp \left(-\tilde{\xi}\left(1, \tilde{\xi}\left(\frac{1}{3}, \lambda\right)\right) L+o(L)\right), &
\end{array}
$$


by the cascade relations (4.1). Comparing with (4.5) gives $\tilde{\xi}\left(1, \tilde{\xi}\left(\frac{1}{3}, \lambda\right)\right)=\tilde{\xi}(1, u(\lambda))$. Finally,

$$
\tilde{\xi}\left(\frac{1}{3}, \lambda\right)=u(\lambda)
$$

follows, since $\lambda^{\prime} \mapsto \tilde{\xi}\left(1, \lambda^{\prime}\right)$ is strictly increasing.

\section{Crossing exponents for critical percolation}

It has been conjectured [42] that $\mathrm{SLE}_{6}$ corresponds to the scaling limit of critical percolation clusters. As additional support for this conjecture, we now show that it implies the conjectured formula for the exponents corresponding to the probability that a long rectangle is crossed by $p$ disjoint paths or clusters of critical percolation ([13], [8], [3]).

Let us first explain the conjectured relation between $\mathrm{SLE}_{6}$ and critical percolation. Let $D \subset \mathbf{C}$ be a domain whose boundary $\partial D \subset \mathbf{C}$ is a simple closed curve. Let $a, b \in \partial D$ be distinct points. Let $\gamma_{1}$ be the counterclockwise arc on $\partial D$ from $a$ to $b$, and let $\gamma_{2}$ be the clockwise arc on $\partial D$ from $a$ to $b$. Let $\delta>0$, and consider a fine hexagonal grid $H$ in the plane with mesh $\delta$; that is, each face of the grid is a regular hexagon with edges of length $\delta$, and each vertex has degree 3 . For simplicity, assume that $\partial D$ does not pass through a vertex of $H$, and that $a$ and $b$ do not lie on edges of $H$. Color each hexagon of $H$ independently, black or white, with probability $\frac{1}{2}$. Then the union of the black hexagons forms one of the standard models for critical percolation (see Grimmett [14] for percolation background and references).

Given the random coloring, there is a unique path $\beta \subset \bar{D}$ that starts at $a$ and ends at $b$, such that whenever $\beta$ is not on $\gamma_{1}$ it has a black hexagon on its "right", and whenever $\beta$ is not on $\gamma_{2}$ it has a white hexagon on its "left". This path is the boundary between the union of the white clusters in $D$ touching $\gamma_{2}$ and the black clusters in $D$ touching $\gamma_{1}$. Let $f: D \rightarrow \mathbf{H}$ be a conformal homeomorphism such that $f(a)=0$ and $f(b)=\infty$, and parameterize $\beta$ in such a way that $A(f(\beta[0, t]))=t$. Let $D_{t}$ be the component of $D \backslash \beta[0, t]$ that has $b$ on its boundary, and let $K_{t}=D \backslash D_{t}$. The conjecture from [42] (stated a bit differently) is that as $\delta \rightarrow 0$ the process $\left(K_{t}, t \geqslant 0\right)$ converges to $\mathrm{SLE}_{6}$ from $a$ to $b$ in $D$. In light of this conjecture, the Locality Theorem 2.2 and its corollaries are very natural.

Now consider an arc $I \subset \partial D$, which contains $b$ but not $a$. Let $b_{1}$ and $b_{2}$ be the endpoints of $I$, labeled in such a way that the triplet $a, b_{1}, b_{2}$ is in counterclockwise order around $D$. Let $\gamma_{1}^{\prime} \subset \gamma_{1}$ be the counterclockwise arc from $a$ to $b_{1}$, and let $\gamma_{2}^{\prime} \subset \gamma_{2}$ be the clockwise arc from $a$ to $b_{2}$. Let $T$ be the first time such that $\beta(t) \in I$, and set $S:=\bigcup_{t<T} K_{t}$. Then the component $\alpha_{1}$ of $\partial S \cap D$ joining $\gamma_{1}^{\prime}$ to $I$ is a crossing in $\overline{\mathcal{B}}$ from $\gamma_{1}^{\prime}$ to $I$ which is "maximal", in the sense that any other crossing $\alpha \subset \overline{\mathcal{B}}$ from $\gamma_{1}^{\prime}$ to $I$ is separated by $\alpha_{1}$ from $b_{2}$ in $D$. 
Let $L$ be large, and recall the definition of the rectangle $\mathcal{R}=\mathcal{R}(L)$ with corners given by (3.1). Let $p \in \mathbf{N}_{+}$and $\sigma=\left(\sigma_{1}, \ldots, \sigma_{p}\right) \in\{\text { black, white }\}^{p}$. Consider the event $C_{\sigma}(\mathcal{R})$ that there are paths $\alpha_{1}, \ldots, \alpha_{p}$ from $\left[A_{4}, A_{1}\right]$ to $\left[A_{2}, A_{3}\right]$ in $\mathcal{R}$ such that each $\alpha_{j}$ is contained in the union of the hexagons of color $\sigma_{j}$, there is no hexagon which intersects more than one of these paths, and $\alpha_{j+1}$ separates $\alpha_{j}$ from $\left[A_{1}, A_{2}\right]$ in $\mathcal{R}$ when $j=1,2, \ldots, p-1$.

Take $\alpha_{1}$ to be the topmost crossing with color $\sigma_{1}$, if such exists, let $\alpha_{2}$ be the topmost crossing with color $\sigma_{2}$ which is below all the hexagons meeting $\alpha_{1}$, etc. Then $C_{\sigma}(\mathcal{R})$ holds if and only if these specific $\alpha_{1}, \ldots, \alpha_{p}$ exist. Note that after we condition on $\alpha_{1}$, the hexagons "below" it are still independent and are black or white with probability $\frac{1}{2}$. Hence the following formula holds:

$$
\mathbf{P}\left[C_{\sigma}(\mathcal{R})\right]=\mathbf{P}\left[\alpha_{1} \text { exists }\right] \mathbf{E}\left[\mathbf{P}\left[C_{\sigma_{+1}}\left(\mathcal{R}_{\alpha_{1}}\right) \mid \alpha_{1}\right] \mid \alpha_{1} \text { exists }\right]
$$

where $\sigma_{+1}=\left(\sigma_{2}, \sigma_{3}, \ldots, \sigma_{p}\right), \mathcal{R}_{\alpha_{1}}$ is the union of the hexagons below $\alpha_{1}$, and $C_{\sigma_{+1}}\left(\mathcal{R}_{\alpha_{1}}\right)$ is the event that there are multiple crossings with colors specified by $\sigma_{+1}$ from $\left[A_{4}, A_{1}\right]$ to $\left[A_{2}, A_{3}\right]$ in $\mathcal{R}_{\alpha_{1}}$.

It is clear that $\mathbf{P}\left[C_{\sigma}(\mathcal{R})\right]$ does not depend on the choice of the sequence $\sigma$, but only its length. Moreover, the conjectured conformal invariance (or the conjecture that $\mathrm{SLE}_{6}$ is the scaling limit) implies that $\lim _{\delta \rightarrow 0} \mathbf{P}\left[C_{\sigma}(\mathcal{D})\right]$ depends on the quadrilateral $\mathcal{D}$ only through its conformal modulus. Hence define

$$
f_{p}(L):=\lim _{\delta \rightarrow 0} \mathbf{P}\left[C_{\sigma}(\mathcal{R}(L))\right], \quad \sigma \in\{\text { black, white }\}^{p} .
$$

We also set $f_{p}(\infty):=0$ and $f_{0}(L):=1_{\{L<\infty\}}$.

Let $S$ be the $\mathrm{SLE}_{6}$-hull from $A_{4}$ to $I:=\left[A_{1}, A_{2}\right] \cup\left[A_{2}, A_{3}\right]$ in $\mathcal{R}=\mathcal{R}(L)$, as defined in $\S 3.1$. Let $\mathcal{R}_{-}$be the component of $\mathcal{R} \backslash S$ which has $A_{1}$ on its boundary, and let $\mathcal{L}$ denote the $\pi$-extremal length from $\left[A_{4}, A_{1}\right]$ to $\left[A_{2}, A_{3}\right]$ in $\mathcal{R}_{-}$. Note that $\mathcal{L}=\infty$ if $\bar{S} \cap\left[A_{1}, A_{2}\right] \neq \varnothing$. Then we have

$$
f_{p}(L)=\mathbf{E}\left[f_{p-1}(\mathcal{L})\right], \quad p=1,2, \ldots
$$

To completely justify this step requires more work, which we omit, since this whole discussion depends on a conjecture anyway. The slight difficulty has to do with the fact that having a crossing of a closed rectangle is a closed condition, and the probability of a closed event can go up when taking a weak limit of measures. One simple way to deal with this is to note that when the continuous process has a crossing in the rectangle $\mathcal{R}(L+\varepsilon)$, every sufficiently close discrete approximation of it has a crossing of the rectangle $\mathcal{R}(L)$.

Consequently, induction and Theorem 3.1 give

$$
f_{p}(L)=\exp \left(-(L+O(1)) v_{p}\right), \quad L \rightarrow \infty
$$


where

$$
v_{p}=u^{\circ p}(0)=\frac{1}{6} p(p+1)
$$

as before. Here, the constant implicit in the $O(1)$-notation may depend on $p$.

Note also that if

$$
\sigma=(\text { white, black, white, black, ..., white }) \in\{\text { black, white }\}^{2 k-1} \text {, }
$$

then (in the discrete setting) the event $C_{\sigma}(\mathcal{R})$ is identical to the event that the rectangle $\mathcal{R}$ is crossed from left to right by $k$ disjoint white clusters.

The exponents (5.2) are those predicted in [8], [3].

\section{References}

[1] Ahlfors, L. V., Conformal Invariants: Topics in Geometric Function Theory. McGrawHill, New York, 1973.

[2] Aizenman, M., The geometry of critical percolation and conformal invariance, in STATPHYS 19 (Xiamen, 1995), pp. 104-120. World Sci. Publishing, River Edge, NJ, 1996.

[3] Aizenman, M., Duplantier, B. \& Aharony, A., Path crossing exponents and the external perimeter in 2D percolation. Phys. Rev. Lett., 83 (1999), 1359-1362.

[4] Belavin, A. A., Polyakov, A. M. \& Zamolodchikov, A. B., Infinite conformal symmetry in two-dimensional quantum field theory. Nuclear Phys. B, 241 (1984), 333-380.

[5] Burdzy, K. \& Lawler, G.F., Non-intersection exponents for Brownian paths. Part I: Existence and an invariance principle. Probab. Theory Related Fields, 84 (1990), 393-410.

[6] Cardy, J. L., Conformal invariance and surface critical behavior. Nuclear Phys. B, 240 (1984), 514-532.

[7] - Critical percolation in finite geometries. J. Phys. A, 25 (1992), L201-L206.

[8] - The number of incipient spanning clusters in two-dimensional percolation. J. Phys. A, 31 (1998), L105.

[9] Cranston, M. \& Mountford, T., An extension of a result of Burdzy and Lawler. Probab. Theory Related Fields, 89 (1991), 487-502.

[10] Duplantier, B., Loop-erased self-avoiding walks in two dimensions: Exact critical exponents and winding numbers. Phys. A, 191 (1992), 516-522.

[11] - Random walks and quantum gravity in two dimensions. Phys. Rev. Lett., 81 (1998), 5489-5492.

[12] Duplantier, B. \& KWON, K.-H., Conformal invariance and intersection of random walks. Phys. Rev. Lett., 61 (1988), 2514-2517.

[13] Duplantier, B. \& Saleur, H., Exact determination of the percolation hull exponent in two dimensions. Phys. Rev. Lett., 58 (1987), 2325-2328.

[14] Grimmett, G., Percolation. Springer-Verlag, New York, 1989.

[15] Ikeda, N. \& Watanabe, S., Stochastic Differential Equations and Diffusion Processes, 2nd edition. North-Holland Math. Library, 24. North-Holland, Amsterdam, 1989.

[16] Kenyon, R., Conformal invariance of domino tiling. Ann. Probab., 28 (2000), 759-795.

[17] - The asymptotic determinant of the discrete Laplacian. Acta Math., 185 (2000), 239-286.

[18] - Long-range properties of spanning trees. Probabilistic techniques in equilibrium and nonequilibrium statistical physics. J. Math. Phys., 41 (2000), 1338-1363. 
[19] Langlands, R., Pouliot, P. \& Saint-Aubin, Y., Conformal invariance in two-dimensional percolation. Bull. Amer. Math. Soc. (N.S.), 30 (1994), 1-61.

[20] LaWler, G. F., Intersections of Random Walks. Birkhäuser Boston, Boston, MA, 1991.

[21] - Hausdorff dimension of cut points for Brownian motion. Electron. J. Probab., 1:2 (1996), 1-20 (electronic).

[22] - The dimension of the frontier of planar Brownian motion. Electron. Comm. Probab., 1:5 (1996), 29-47 (electronic).

[23] - The frontier of a Brownian path is multifractal. Preprint, 1997.

[24] Lawler, G.F. \& Puckette, E. E., The intersection exponent for simple random walk. Combin. Probab. Comput., 9 (2000), 441-464.

[25] Lawler, G.F., Schramm, O. \& Werner, W., Values of Brownian intersection exponents, II: Plane exponents. Acta Math., 187 (2001), 275-308. http://arxiv.org/abs/math.PR/0003156.

[26] - Values of Brownian intersection exponents, III: Two-sided exponents. To appear in Ann. Inst. H. Poincaré Probab. Statist. http://arxiv.org/abs/math.PR/0005294.

[27] - Analyticity of intersection exponents for planar Brownian motion. To appear in Acta Math., 188 (2002). http://arxiv.org/abs/math.PR/0005295.

[28] LAWLER, G. F. \& WERnER, W., Intersection exponents for planar Brownian motion. Ann. Probab., 27 (1999), 1601-1642.

[29] - Universality for conformally invariant intersection exponents. J. Eur. Math. Soc. (JEMS), 2 (2000), 291-328.

[30] Lebedev, N. N., Special Functions and Their Applications. Dover, New York, 1972.

[31] Lehto, O. \& Virtanen, K. I., Quasiconformal Mappings in the Plane, 2nd edition. Grundlehren Math. Wiss., 126. Springer-Verlag, New York-Heidelberg, 1973.

[32] LöwnER, K., Untersuchungen über schlichte konforme Abbildungen des Einheitskreises, I. Math. Ann., 89 (1923), 103-121.

[33] Madras, N. \& Slade, G., The Self-Avoiding Walk. Birkhäuser Boston, Boston, MA, 1993.

[34] Majumdar, S. N., Exact fractal dimension of the loop-erased random walk in two dimensions. Phys. Rev. Lett., 68 (1992), 2329-2331.

[35] Mandelbrot, B. B., The Fractal Geometry of Nature. Freeman, San Francisco, CA, 1982.

[36] Marshall, D.E. \& Rohde, S., The Loewner differential equation and slit mappings. Preprint.

[37] Pommerenke, CH., On the Loewner differential equation. Michigan Math. J., 13 (1966), $435-443$.

[38] - Boundary Behaviour of Conformal Maps. Grundlehren Math. Wiss., 299. SpringerVerlag, Berlin, 1992.

[39] Revuz, D.\& Yor, M., Continuous Martingales and Brownian Motion. Grundlehren Math. Wiss., 293. Springer-Verlag, Berlin, 1991.

[40] Rohde, S. \& Schramm, O., Basic properties of SLE. Preprint.

[41] Rudin, W., Real and Complex Analysis, 3rd edition. McGraw-Hill, New York, 1987.

[42] Schramm, O., Scaling limits of loop-erased random walks and uniform spanning trees. Israel J. Math., 118 (2000), 221-288. 
Gregory F. LAWLer

Department of Mathematics

Duke University

Box 90320

Durham, NC 27708-0320

U.S.A.

jose@math.duke.edu

WENDELIN WERNER

Département de Mathématiques

Université Paris-Sud

Bât. 425

FR-91405 Orsay Cedex

France

wendelin.werner@math.u-psud.fr

Received January 3, 2000

\author{
Oded Schramm \\ Microsoft Research \\ 1, Microsoft Way \\ Redmond, WA 98052 \\ U.S.A. \\ schramm@microsoft.com
}

